\title{
Extended hesitant fuzzy linguistic term sets and their aggregation in group decision making
}

\author{
Hai Wang* \\ School of Economics and Management, Southeast University \\ Nanjing, Jiangsu 211189, China
}

Received 15 March 2014

Accepted 17 March 2014

\begin{abstract}
Group decision making problems which organize a group of experts to evaluate a set of alternatives with respect to several criteria are commonly discussed recently. Hesitant fuzzy linguistic term sets, characterized by a set of consecutive linguistic terms, act as a new model for qualitative settings where experts think of several possible linguistic values or richer expressions than a single term. When evaluating an indicator, alternative or variable in group decision making, however, linguistic terms involved in an expression derived by the group may be not always consecutive. Therefore, we generalize hesitant fuzzy linguistic term sets by enabling any non-consecutive linguistic terms in them, and refer to as extended hesitant fuzzy linguistic term sets (EHFLTSs). EHFLTSs can be constructed by the union of hesitant fuzzy linguistic term sets given by individual expert. As owning more desirable mathematical properties, EHFLTSs are flexible for develop complex decision model. Some basic operation and envelop of EHFLTSs are defined and some mathematical properties are discussed as well. For the sake of application in group decision making, we develop two classes of aggregation operators for aggregating a set of EHFLTSs to suit the cases where weighting vectors take the form of real numbers and linguistic terms respectively. Then a new group decision making model is formed and corresponding processes for two distinct scenarios are developed. A practical application clarifies the rationality and advantages of the proposed technique.
\end{abstract}

Keywords: Group decision making; Hesitant fuzzy set; Hesitant fuzzy linguistic term set; Extended hesitant fuzzy linguistic term set; Aggregation operator.

\section{Introduction}

Decision making by individual and group of individuals, such as committees, governing bodies, juries, business partners, teams, and families, is referred to as individual decision making and group decision making (GDM), respectively. Group decision making is a type of participatory procedure in which multi decision makers (DMs) acting collectively, consider and evaluate alternative courses of action, and select among the alternatives a solution or solutions. Along with the increasing complexity of real world decision making problems, uncertainties are unavoidable but quite challenging to modeling. To cope with such uncertainties, several tools have been developed, such as evidential reasoning theories ${ }^{1-2}$ in probabilistic setting and fuzzy sets (Z-FSs) ${ }^{3}$ theories in imprecise or vague setting. The latter has been successfully applied to handle kinds of fuzzy information. Recently, some extensions and generalizations of fuzzy sets have been proposed to solve complex problems. These extensions can be concluded as the following two classes. Extensions of the first class are suitable for quantitative

${ }^{*}$ Corresponding author. E-mail address: wanghai17@sina.com 
situations, such as type- 2 fuzzy sets ${ }^{4}$, type-n fuzzy sets ${ }^{5}$, intuitionistic fuzzy sets ${ }^{6}$ or interval-value fuzzy sets, fuzzy multisets ${ }^{7}$, hesitant fuzzy sets (HFSs) ${ }^{8}$ and generalized hesitant fuzzy sets ${ }^{9}$. In many situations, however, the information can only be assessed in a qualitative form instead of a quantitative one. For example, when evaluating the degree of comfort of a car, "good", "poor" may be used ${ }^{10}$. Thus the second class focuses on fuzzy linguistic approaches regarding computing with words (CWW). Since Zadeh ${ }^{11}$ had presented the concept of linguistic variables, several linguistic models were extended, such as the linguistic model based on membership functions ${ }^{12}$, the linguistic model based on type-2 fuzzy sets ${ }^{13}$, the linguistic model based on ordinal scales ${ }^{14-16}$, the linguistic 2-tuple model ${ }^{17}$, the proportional 2-tuple model ${ }^{18}$ and so on.

As the base of linguistic computation, linguistic representation models are quite limited. Most of the existing techniques usually take use of single and very simple linguistic term to represent the information presented by experts. In practical, however, an expert may think of more than one term at the same time and look forward to a more complex linguistic term, instead of a single term, to represent his/her evaluations about problems defined under uncertainty. With this view, Rodriguez et al. ${ }^{19}$ presented the concept of hesitant fuzzy linguistic term set (HFLTS) by the idea of HFS in 2012. Based on a predefined linguistic term set, HFLTS is several consecutive ordered linguistic terms of the set. For example, the degree of comfort of a car is evaluated by the linguistic term set \{"very good", "good", "poor"\}. One's evaluation may be "at least good". The evaluation can be represented by a HFLTS \{"very good", "good"\} and can be seen as a generalized linguistic term "very good" or "good". It is obvious that HFLTS is a powerful tool for one expert to express his her assessment by either a single linguistic term or complex linguistic terms.

Recently, Rodriguez et al. ${ }^{20}$ used HFLTSs to present a GDM approach dealing with comparative linguistic expressions. In this study, experts' preferences are expressed by HFLTS. The envelope of each HFLTS, i.e. a linguistic interval, is calculated before aggregating experts' preferences by proper operators. It is rational and accurate if the linguistic terms in HFLTSs are consecutive. However, it may be not suitable for some more complex GDM situations. In individual decision making, an expert's evaluation, relative measure and absolute measure, is represented by one term or several consecutive terms according to his/her preference. When it comes to GDM, individual evaluations can be represented by HFLTSs, but the group's evaluations can not be always represented by them. Suppose a decision organization with three groups of experts is authorized to assess the satisfactory degree of an alternative with respect to a criterion by a linguistic term set \{ "very good", "good", "indifferent", "bad", "very bad"\}. In Group 1, some experts provide "very good" surely, others provide "good" without hesitancy, and thus the assessment can be represented by a HFLTS \{"very good", "good"\}. While in Group 2, some experts provide "bad" doubtless, others insist on at least "good". Thus three linguistic terms are considered in this assessment, i.e. "very good", "good" and "bad". We can not use a HFLTS to represent it as linguistic terms are not consecutive here. Group 3 provides between "bad" and "good" consistently, which result to a HFLTS \{"good", "indifferent", "bad"\}. An alternative resolution of this problem is that aggregating, by proper aggregating operators, as in $\mathrm{Xu}{ }^{10}$, the linguistic information within each group at first and then aggregating the resultant information among groups because we can not represent the assessment of Group 2 by any existing linguistic term set. Therefore, there are at least three steps of aggregations at different levels if multi criteria are taken into account in the problem. Elimination of the aggregation within group by considering all the possible linguistic terms is meaningful during the decision making process. Take the assessment of Group 3 for example, original information is "good", "indifferent" or "bad". If the linguistic averaging operator ${ }^{21}$ is used, the assessment may result to "indifferent"; if some weights are further considered, some virtual linguistic terms ${ }^{16}$ may be derived. Comparing to the original assessment, we lose some important information at the beginning of the decision making process. Another alternative resolution is the evidential reasoning algorithm ${ }^{2}$ if corresponding probabilities are provided associated with the linguistic terms. For example, if weights of experts in Group 1 are equal, one half of experts support "very good", others support "good", the assessment of Group 1 may be expressed as ("“very good", 0.5), ("good", 0.5), ("indifferent", 0), ("bad", 0), ( "very bad", 0)\}. By the 
evidential reasoning algorithm, the overall assessment of the alternative is expressed by a probabilistic distribution as well. But in practical, we may just have hesitancy on some linguistic terms while not be sure about the probabilistic distribution. Further, ordinal terms are usually used as in this problem. But the evidential reasoning algorithm does not consider the ordinal relation of the linguistic terms at all. In conclusion, when evaluating the satisfactory degree by linguistic terms, the difficulty is not because we have some probabilistic distributions on the possible linguistic terms, but because we have a set of consecutive or nonconsecutive possible linguistic terms. It is useful to deal with all the possible linguistic terms rather than considering just an aggregation operator.

Therefore, in this study, we propose a new linguistic term model named EHFLTS motivated by the idea of HFSs for linguistic GDM setting. An EHFLTS is a subset of a pre-defined linguistic term set. The linguistic information involved in an EHFLTS is considered as a generalized linguistic term, referred to as extended hesitant fuzzy linguistic term (EHFLT). We develop some basic operations for EHFLTSs and some arithmetic operations for EHFLTs. Two classes of aggregation operators with distinct forms of weighting vector are also developed for fusing a set of EHFLTs. A linguistic GDM model based on the proposed EHFLTSs, associated with two specified processes, is presented for potential application. The main advantages of the proposed EHFLTSs are as follow. First, theoretically, EHFLTSs can represent linguistic assessments with consecutive and nonconsecutive linguistic terms, thus all the possible linguistic terms are taken into account without a pre-aggregation process in GDM. Comparing to existing linguistic decision making model, we eliminate at least one aggregation procedure. Second, the probabilistic distribution is not necessary when evaluating. Thus experts can express their evaluations with flexible forms while no extra work is needed. At last, as a generalization of Rodriguez's HFLTSs, the proposed EHFLTSs own better mathematical properties. For example, the union, intersection and complement of EHFLTSs are closed.

To achieve it, the structure of the paper is as follows. Section 2 reviews some related preliminaries, such as some fuzzy linguistic models, HFSs and HFLTSs. In Section 3, EHFLTSs and EHFLTs are defined, some basic operations associated with their relationships are discussed, and comparison laws are developed as well. Section 4 presents the extension principle and some specific aggregation operators. Section 5 develops the EHFLTSs-based linguistic GDM model and specifies two processes in different scenarios. A practical application is presented in Section 6, as well as comparison with an existing method. Then Section 7 concludes the paper.

\section{Preliminaries}

Due to the proposal of utilizing HFSs to generalize traditional fuzzy linguistic label sets, this section is devoted to recall some preliminaries involved in fuzzy linguistic approach, HFSs and HFLTSs.

\subsection{Fuzzy linguistic models}

In many real-world situations, the use of linguistic information is very straightforward and suitable to express the satisfaction associated with an outcome and a state of nature ${ }^{10,19,22}$. Fuzzy linguistic approaches ${ }^{23-25}$ are used to model the linguistic information and the fuzzy set theory ${ }^{3}$ is utilized to manage the uncertainties. For convenience, let $\tilde{S}=\left\{s_{i} \mid i=0,1, \cdots, g\right\}$ be a finite and totally ordered discrete linguistic term set, where $g$ is a positive even integer, $s_{i}$ represents a possible value for a linguistic variable such that ${ }^{26}$ :

(1) The set is ordered: $s_{i} \geq s_{j}$ iff $i \geq j$;

(2) The negation operator is defined: $\operatorname{neg}\left(s_{i}\right)=s_{j}$ such that $j=g-i$.

The cardinality of $\tilde{S}$ is odd, and should be neither too small nor too rich ${ }^{27}$.

Example $1^{28}$. A set of seven linguistic terms, could be:

$\tilde{S}=\left\{s_{0}=\right.$ none, $s_{1}=$ very low, $s_{2}=$ low, $s_{3}=$ medium, $s_{4}=$ high, $s_{5}=$ very high, $s_{6}=$ perfect $\}$.

Further, Fig. 1 shows $\tilde{S}$ with the syntax and semantics of the seven terms.

In decision making process, linguistic terms are usually used and computed directly ${ }^{16,29-30}$. But it is not easy to define some intuitive operation laws for the above kind of linguistic term set. Therefore, $\mathrm{Xu}{ }^{29}$ redefined the linguistic term set $\tilde{S}$ by another form

$$
S=\left\{s_{i} \mid i=-t, \cdots, t\right\},
$$


where $t$ is a positive integer, $s_{i}$ owns the following characteristics:

(1) The set is ordered: $s_{i}>s_{j}$ iff $i>j$;

(2) The negation operator is defined: $\operatorname{neg}\left(s_{i}\right)=s_{-i}$, especially $\operatorname{neg}\left(s_{0}\right)=s_{0}$.

Example 2 . In this case, a set of seven linguistic terms, could be:

$S=\left\{s_{-3}=\right.$ very poor, $s_{-2}=$ poor,$s_{-1}=$ slightly poor, $s_{0}=$ fair, $s_{1}=$ slightly good, $s_{2}=$ good, $s_{3}=$ very $\left.\operatorname{good}\right\}$.

Obviously, $s_{0}$ represents an evaluation of "indifference" ${ }^{10}$. Given a discrete term set with the latter form $S, \mathrm{Xu}^{29}$ further extended it to a continuous term set

$$
\bar{S}=\left\{s_{\alpha} \mid \alpha \in[-q, q]\right\}
$$

to preserve all given information, where $q(q>t)$ is a sufficiently large positive integer. If $s_{\alpha} \in S$, as seen in Fig. 2, $s_{\alpha}$ is called an original linguistic term; otherwise, $s_{\alpha}$ is a virtual linguistic term. In general, original linguistic terms are used for experts to conduct evaluations, and virtual linguistic terms only appear in calculations. To accomplish processes of $\mathrm{CW}$ with this representation, the following operation laws are introduced.

Definition $1^{21}$. Let $s_{\alpha}, s_{\beta} \in \bar{S}$ be any two linguistic terms and $\lambda, \lambda_{1}, \lambda_{2} \in[0,1]$, then

(1) $s_{\alpha} \oplus s_{\beta}=s_{\alpha+\beta}$;

(2) $s_{\alpha} \otimes s_{\beta}=s_{\alpha \beta}$;

(3) $\lambda s_{\alpha}=s_{\lambda \alpha}$;

(4) $\left(s_{\alpha}\right)^{\lambda}=s_{\alpha^{\lambda}}$.

By the operation laws in Definition 1, we can see the representation $S=\left\{s_{i} \mid i=-t, \cdots, t\right\}$ is more in accord with actual situations than the former representation $\tilde{S}=\left\{s_{i} \mid i=0,1, \cdots, g\right\}$. In fact, let's consider the two special linguistic term sets hereinabove. We have $s_{-2} \oplus s_{2}=s_{0}$ in $S$ of Example 2, which means "poor" and "good" result to "fair". While in $\tilde{S}$ of Example 1, we have $s_{2} \oplus s_{4}=s_{6}$, which means "low" and "high" become "perfect". However, it should be note that the results of this symbolic computational model are usually virtual linguistic terms and thus may be out of the universe of discourse of the linguistic variable ${ }^{31}$. In

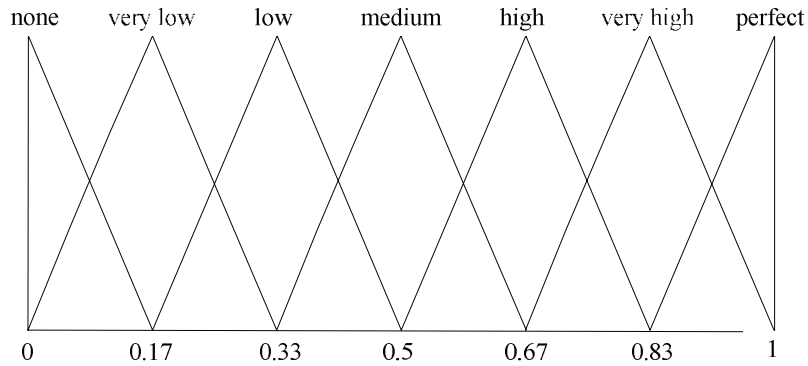

Fig. 1. A set of seven terms with its semantics.

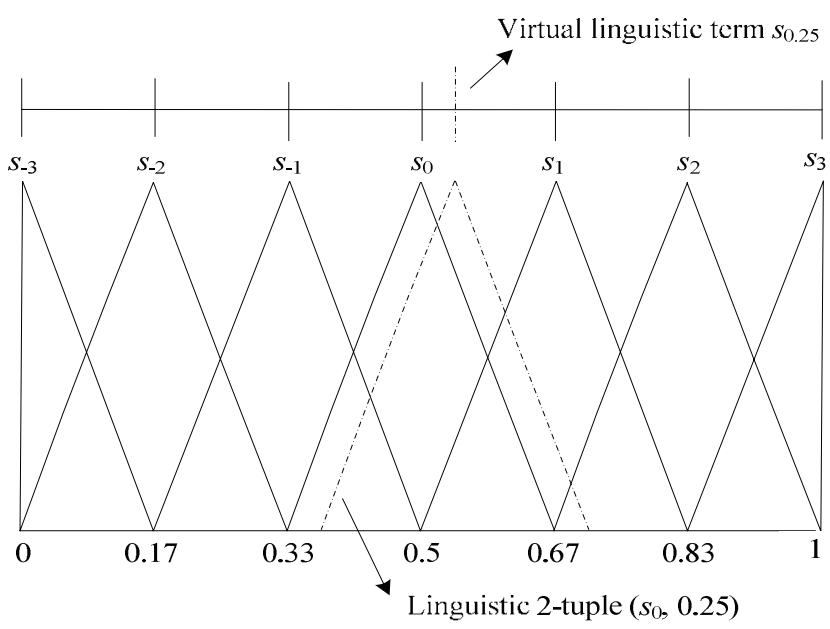

Fig. 2 Example of the virtual linguistic terms and linguistic 2tuple.

addition, this model does not use semantics or syntax which leads to the result not interpretable ${ }^{32}$.

Serving as another accurate linguistic model, the linguistic 2-tuple model ${ }^{17}$ keeps a syntax and fuzzy semantics in its representation. Let $S=\left\{s_{i} \mid i=-t, \cdots, t\right\}$ be a linguistic term set and $\alpha \in[-t, t]$ a value representing the result of a symbolic aggregation operation, then the 2-tuple that expresses the equivalent information to $\alpha$ is obtained by the following function:

$$
\begin{aligned}
& \Delta:[-t, t] \rightarrow S \times[-0.5,0.5) \\
& \Delta(\alpha)=\left(s_{i}, x\right), \quad \text { with }\left\{\begin{array}{l}
s_{i}, i=\operatorname{round}(\alpha) \\
x=\alpha-i, x \in[-0.5,0.5)
\end{array}\right.
\end{aligned}
$$

Let $\left(s_{i}, x\right)$ be a linguistic 2-tuple associated with $S=\left\{s_{i} \mid i=-t, \cdots, t\right\}$. There is always a function $\Delta^{-1}$ such that, from a linguistic 2-tuple, it returns its equivalent numerical value $\alpha \in[-t, t]^{31}$. As can be seen in Fig. 2, the linguistic 2-tuple model keeps the fuzzy representation and syntax. 
We can also find in Fig. 2 that there is an interesting relationship between the linguistic virtual model and the linguistic 2-tuple model (See Dong et al. ${ }^{33}$ for detail). Given $S=\left\{s_{i} \mid i=-t, \cdots, t\right\}$, if $\alpha \in[-t, t]$, then there is a one to one mapping between virtual term $s_{\alpha}$ and 2-tuple $\left(s_{i}, x\right)$, such that $\alpha=i+x$. As will be illustrated in Section 4.3, lower indices of virtual terms involved in this study are bounded in $[-t, t]$. We use the linguistic virtual model for the procedure of computing hereinafter, and then translate the resultant virtual term into 2-tuple to show its linguistic representation.

\subsection{HFSs and HFLTSS}

Sometimes, it is difficult to determine the membership of an element into a fixed set and which may be caused by a doubt among a set of different values. For the sake of a better description of this situation, Torra introduced the concept of HFSs as a generalization of fuzzy sets as follow.

Definition $2^{8}$. Let $X$ be a fixed set, then a hesitant fuzzy set (HFS) on $X$ in terms of a function $h$ is that when applied to $X$ returns a subset of $[0,1]$.

Furthermore, given a set of fuzzy sets, a HFS could be defined in accordance with the union of their memberships.

Definition $3^{8}$. Given a set of $N$ membership functions: $M=\left\{\gamma_{1}, \cdots, \gamma_{N}\right\}$, the HFS associated with $M$, that is $h_{M}$, is defined as follow:

$$
h_{M}(x)=\bigcup_{\gamma \in M}\{\gamma(x)\} .
$$

For a given $x$ in $X, h_{M}(x)$ is a set of some values in $[0,1]$, denoting the possible memberships of $x$ to a set. To be easily understood, Xia and $\mathrm{Xu}^{34}$ expressed the HFS by a mathematical symbol $\left\{\left\langle x, h_{M}(x)\right\rangle \mid x \in X\right\}$, called $h=h_{M}(x)$ a hesitant fuzzy element (HFE) and $H$ the set of all HFEs.

When decision information is represented by a collection of HFSs, it is necessary to introduce a function or mechanism to aggregate them for final decision making. Torra and Narukawa ${ }^{35}$ proposed an extension principle which permits us to export operations on Z-FSs to T-HFSs as follow.
Definition $4{ }^{35}$. Let $\Theta$ be a function $\Theta:[0,1]^{N} \rightarrow[0,1]$, $H=\left\{h_{1}, h_{2}, \cdots h_{N}\right\}$ be a set of HFSs on the reference set $X$. Then the extension of $\Theta$ on $H$ is defined for each $x$ in $X$ by:

$$
\Theta_{H}(x)=\bigcup_{\gamma \in\left\{h_{1}(x) \times h_{2}(x) \times \cdots \times h_{N}(x)\right\}}\{\Theta(\gamma)\} .
$$

Bearing in mind the idea of fuzzy linguistic approaches and HFSs, Rodriguez et al. ${ }^{19}$ presented the following concept of HFLTSs.

Definition $5^{19}$. Let $S$ be a linguistic term set, then a HFLTS, $\tilde{H}_{S}$, is an ordered finite subset of consecutive linguistic terms of $S$.

Three basic operations of HFLTS are defined as follows.

Definition $6{ }^{19}$. Let $\tilde{H}_{S}, \tilde{H}_{S}^{1}$ and $\tilde{H}_{S}^{2}$ be three HFLTSS, then the following operations are defined:

(1) Complement:

$$
\tilde{H}_{S}^{c}=S-\tilde{H}_{S}=\left\{s_{i} \mid s_{i} \in S, s_{i} \notin \tilde{H}_{S}\right\} ;
$$

(2) Union

$$
\tilde{H}_{S}^{1} \cup \tilde{H}_{S}^{2}=\left\{s_{i} \mid s_{i} \in \tilde{H}_{S}^{1} \quad \text { or } \quad s_{i} \in \tilde{H}_{S}^{2}\right\} ;
$$

(3) Intersection:

$$
\tilde{H}_{S}^{1} \cap \tilde{H}_{S}^{2}=\left\{s_{i} \mid s_{i} \in \tilde{H}_{S}^{1} \quad \text { and } \quad s_{i} \in \tilde{H}_{S}^{2}\right\} .
$$

Note that the union of two HFLTSs may be not close. If the linguistic terms set $S$ in Example 2 is used, let $\tilde{H}_{S}^{1}=\left\{s_{0}\right\}$ and $\tilde{H}_{S}^{1}=\left\{s_{2}, s_{3}\right\}$, then the union of these two HFLTSs is $\left\{s_{0}, s_{2}, s_{3}\right\}$. Thus the result is not consecutive any more.

\section{Extended hesitant fuzzy linguistic term sets}

We present the concept of EHFLTSs as well as some basic operation in this section. Then some mathematical properties are discussed.

\subsection{Definition of EHFLTSS}

As discussed in Introduction, we may have a doubt among several possible linguistic terms when considering the degree of an alternative satisfying a certain criterion in GDM problems. In order to handle this kind of assessment in decision making process directly, instead of preparatory aggregation, we extend the concept of HFLTSs. Let's begin with an example. 
Example 3. Two groups of experts are authorized to evaluate a car company in isolation using the linguistic sets $S$ in Example 2. Caused by their different knowledge, experiences and backgrounds, experts of Group 1 provide "at least good", some experts of Group 2 insist on "at least good" as well, while others argue "slightly poor", they can't persuade each other. The assessment of Group 1 can be expressed by a HFLTS as follows,

$$
\tilde{H}_{S}(x)=\left\{s_{2}, s_{3}\right\}
$$

But linguistic terms emerged form Group 2 are not consecutive subset of $S$, thus can't be represented by HFLTS.

To accommodate this kind of uncertain circumstance, we present the following definition.

Definition 7. Let $S$ be a linguistic term set, then an ordered subset of linguistic terms of $S$, that is,

$$
H_{S}(x)=\left\{s_{i} \mid s_{i} \in S\right\},
$$

is called an extended hesitant fuzzy linguistic term set (EHFLTS).

Given a linguistic term set $S=\left\{s_{\alpha} \mid \alpha=-t, \cdots, t\right\}$, the empty EHFLTS and the full EHFTLS for a linguistic variable, $x$, are as follows:

Empty EHFLTS: $H_{S}(x)=\{\}$,

Full EHFLTS: $H_{S}(x)=S$

Similarly, the evaluation of Group 2 in Example 3 can be represented by an EHFLTS:

$$
H_{S}(x)=\left\{s_{-1}, s_{2}, s_{3}\right\}
$$

It is clear that HFLTS is the special case of EHFLTS

In the process of information computing, as we will see hereinafter, virtual linguistic terms are used. Thus for the convenience information processing, an ordered finite subset of virtual linguistic terms set $\bar{S}$ is referred to as EHFLTS as well.

We can see that, usually, an EHFLTS represents one complex evaluation with uncertainties. In this paper, the linguistic terms that appear in an EHFLTS are considered as a generalized linguistic term. Formally, let $X$ be a fixed set, for $x \in X, H_{S}(x)$ can be represented as the following 2-tuple:

$$
\left\{<x, h_{S}(x)>\mid x \in X\right\}
$$

where $h_{S}(x)$ is a set of $p$ linguistic terms, in $S$ (or $\bar{S})$, i.e. $h_{S}(x)=\left\{s_{i_{1}}, s_{i_{2}}, \cdots, s_{i_{p}}\right\}$. For a given $x, h_{S}(x)$ is abbreviated as $h_{S}$, represents all possible linguistic terms, thus is referred to as extended hesitant fuzzy linguistic term (EHFLT). For simplicity, single linguistic term is considered as an EHFLT.

\subsection{Basic operations}

Similar to Rodriguez et al. ${ }^{19}$, we define some basic concept and basic operations of EHFLTSs hereinafter.

For a given EHFLTS $H_{S}(x)$, its upper and lower bound are denoted by:

(1) upper bound: $H_{S}^{+}=\max \left\{s_{i}\right\}=s_{j}, s_{i} \in H_{S}$ and $s_{i} \leq s_{j}$ for any $i$

(2) lower bound: $H_{S}^{-}=\min \left\{s_{i}\right\}=s_{j}, s_{i} \in H_{S}$ and $s_{i} \geq s_{j}$ for any $i$.

Obviously, $H_{S}^{-}$and $H_{S}^{+}$define an uncertain linguistic variable, which simultaneously form the envelope of the EHFLTS. We describe it in the following definition.

Definition 8. Given a EHFLTS $H_{S}(x)$, its envelope, $A_{e n v}\left(H_{S}\right)$, is defined by an uncertain linguistic variable represented by $\left[H_{S}^{-}, H_{S}^{+}\right]$.

Three basic operations are defined straightforward.

Definition 9. Given three EHFLTSs $H_{S}(x), H_{S}^{1}(x)$ and $H_{S}^{2}(x)$ for given $x$ in $X$, then the following operations are defined:

(1) Complement:

$H_{S}^{c}(x)=S-H_{S}(x)=\left\{s_{i} \mid s_{i} \in S, s_{i} \notin H_{S}\right\}$;

(2) Union:

$\left(H_{S}^{1} \cup H_{S}^{2}\right)(x)=\left\{s_{i} \mid s_{i} \in H_{S}^{1}(x) \quad\right.$ or $\left.\quad s_{i} \in H_{S}^{2}(x)\right\}$;

(3) Intersection:

$\left(H_{S}^{1} \cap H_{S}^{2}\right)(x)=\left\{s_{i} \mid s_{i} \in H_{S}^{1}(x)\right.$ and $\left.s_{i} \in H_{S}^{2}(x)\right\}$.

We can see that the results of complement, union and intersection are EHFLTSs as well. According to the union of EHFLTSs, we can draw the following conclusion.

Theorem 1. (Construction axiom) The union of HFLTSS results to EHFLTS 
Proof of Theorem 1 is omitted. We shall note that this construction axiom show potential application of the presented EHFLTSs. Individuals provide their evaluation information by HFLTSs. And then the group's evaluation is formed by the union of these HFLTSs, which results to an EHFLTS. In this procedure, we keep all possible linguistic terms rather than preparatory aggregation. Furthermore, it is very useful when individual priorities are absolutely unknown, for example, in anonymous setting.

For the purpose of aggregation, we further define some arithmetic operations for EHFLTs based on virtual linguistic terms set $\bar{S}$. Note that $\bar{S}$ contains as many $s_{i}$ (original linguistic term or virtual linguistic term) as necessary.

Definition 10. Given three EHFLTs $h_{S}, h_{S}^{1}$ and $h_{S}^{2}$, $\lambda \geq 0$, then:
(1) $h_{S}^{1} \oplus h_{S}^{2}=\bigcup_{s_{i} \in h_{S}^{1}, s_{j} \in h_{S}^{2}}\left\{s_{i} \oplus s_{j}\right\}=\bigcup_{s_{i} \in h_{S}^{1}, s_{j} \in h_{S}^{2}}\left\{s_{i+j}\right\}$;
(2) $h_{S}^{1} \otimes h_{S}^{2}=\bigcup_{s_{i} \in h_{S}^{1}, s_{j} \in h_{S}^{2}}\left\{s_{i} \otimes s_{j}\right\}=\bigcup_{s_{i} \in h_{S}^{1}, s_{j} \in h_{S}}\left\{s_{i j}\right\}$;
(3) $\lambda h_{S}=\bigcup_{s_{i} \in h_{S}}\left\{\lambda s_{i}\right\}=\bigcup_{s_{i} \in h_{S}}\left\{s_{\lambda_{i}}\right\}$;
(4) $\left(h_{S}\right)^{\lambda}=\bigcup_{s_{i} \in h_{S}}\left\{\left(s_{i}\right)^{\lambda}\right\}=\bigcup_{s_{i} \in h_{S}}\left\{s_{i^{\lambda}}\right\}$;
(5) $h_{S}^{1} \vee h_{S}^{2}=\bigcup_{s_{i} \in h_{s}^{1}, s_{j} \in h_{S}^{2}}\left\{\max \left\{s_{i}, s_{j}\right\}\right\}$;
(6) $h_{S}^{1} \wedge h_{S}^{2}=\bigcup_{s_{i} \in h_{S}^{1}, s_{j} \in h_{S}^{2}}\left\{\min \left\{s_{i}, s_{j}\right\}\right\}$.

Example 4. Let $S$ be the linguistic term set in Example 2. $h_{S}^{1}=\left\{s_{-1}, s_{2}\right\}, h_{S}^{2}=\left\{s_{2}, s_{3}, s_{5}\right\}$, then:

$$
\begin{aligned}
h_{S}^{1} \oplus h_{S}^{2}= & \left\{s_{-1} \oplus s_{2}\right\} \bigcup\left\{s_{-1} \oplus s_{3}\right\} \bigcup\left\{s_{-1} \oplus s_{5}\right\} \\
& \bigcup\left\{s_{2} \oplus s_{2}\right\} \bigcup\left\{s_{2} \oplus s_{3}\right\} \bigcup\left\{s_{2} \oplus s_{5}\right\} \\
= & \left\{s_{1}\right\} \bigcup\left\{s_{2}\right\} \bigcup\left\{s_{4}\right\} \bigcup\left\{s_{4}\right\} \bigcup\left\{s_{5}\right\} \bigcup\left\{s_{7}\right\} \\
= & \left\{s_{1}, s_{2}, s_{4}, s_{5}, s_{7}\right\} .
\end{aligned}
$$

\subsection{Properties}

In this section, we will discuss some mathematical properties of the operations defined hereinabove.

Theorem 2. Let $H_{S}(x), H_{S}^{1}(x), H_{S}^{2}(x)$ and $H_{S}^{3}(x)$ be four EHFLTSS for given $x$ in $X$, then

(1) Involutive: $\left(H_{S}^{c}(x)\right)^{c}=H_{S}(x)$;

(2) Commutativity: $\left(H_{S}^{1} \cup H_{S}^{2}\right)(x)=\left(H_{S}^{2} \cup H_{S}^{1}\right)(x)$,

(3) Associative:

$$
\begin{aligned}
& H_{S}^{1}(x) \cup\left(\left(H_{S}^{2} \cup H_{S}^{3}\right)(x)\right)=\left(\left(H_{S}^{1} \cup H_{S}^{2}\right)(x)\right) \cup H_{S}^{3}(x), \\
& H_{S}^{1}(x) \cap\left(\left(H_{S}^{2} \cap H_{S}^{3}\right)(x)\right)=\left(\left(H_{S}^{1} \cap H_{S}^{2}\right)(x)\right) \cap H_{S}^{3}(x) ; \\
& \text { (4) Distributive: } \\
& H_{S}^{1}(x) \cap\left(\left(H_{S}^{2} \cup H_{S}^{3}\right)(x)\right) \\
& \quad=\left(\left(H_{S}^{1} \cap H_{S}^{2}\right)(x)\right) \bigcup\left(\left(H_{S}^{1} \cap H_{S}^{3}\right)(x)\right), \\
& H_{S}^{1}(x) \bigcup\left(\left(H_{S}^{2} \cap H_{S}^{3}\right)(x)\right) \\
& \quad=\left(\left(H_{S}^{1} \bigcup H_{S}^{2}\right)(x)\right) \cap\left(\left(H_{S}^{1} \cup H_{S}^{3}\right)(x)\right) .
\end{aligned}
$$

We can prove Theorem 2 using the same method of Rodriguez et al. ${ }^{19}$. Thus the procedure of proof is omitted.

Furthermore, we will present some properties of operations on EHFLTs, such as commutativity, associative and distributive. The commutativity is given at first.

Theorem 3. (Commutativity) Let $h_{S}^{1}$ and $h_{S}^{2}$ be two EHFLTS, then
(1) $h_{s}^{1} \oplus h_{s}^{2}=h_{s}^{2} \oplus h_{s}^{1}$;
(2) $h_{S}^{1} \otimes h_{S}^{2}=h_{S}^{2} \otimes h_{S}^{1}$;
(3) $h_{S}^{1} \vee h_{S}^{2}=h_{S}^{2} \vee h_{S}^{1}$;
(4) $h_{S}^{1} \wedge h_{S}^{2}=h_{S}^{2} \wedge h_{S}^{1}$.

Proof.

(1) $\begin{aligned} & h_{S}^{1} \oplus h_{S}^{2}=\bigcup_{s_{i} \in h_{S}^{1}, s_{j} \in h_{S}^{2}}\left\{s_{i} \oplus s_{j}\right\}=\bigcup_{s_{i} \in h_{S}^{1}, s_{j} \in h_{S}^{2}}\left\{s_{i+j}\right\} \\ = & \bigcup_{s_{j} \in h_{S}^{2}, s_{i} \in h_{S}^{1}}\left\{s_{j+i}\right\}=\bigcup_{s_{j} \in h_{S}^{2}, s_{i} \in h_{S}^{1}}\left\{s_{j} \oplus s_{i}\right\}=h_{S}^{2} \oplus h_{S}^{1} ;\end{aligned}$

(2) $h_{S}^{1} \otimes h_{S}^{2}=\bigcup_{s_{i} \in h_{S}^{1}, s_{j} \in h_{S}^{2}}\left\{s_{i} \otimes s_{j}\right\}=\bigcup_{s_{i} \in h_{S}^{1}, s_{j} \in h_{S}^{2}}\left\{s_{i j}\right\}$

$$
=\bigcup_{s_{j} \in h_{S}^{2}, s_{i} \in h_{S}^{1}}\left\{s_{j i}\right\}=\bigcup_{s_{j} \in h_{S}^{2}, s_{i} \in h_{S}^{1}}\left\{s_{j} \otimes s_{i}\right\}=h_{S}^{2} \otimes h_{S}^{1} ;
$$

(3) $h_{S}^{1} \vee h_{S}^{2}=\bigcup_{s_{i} \in h_{S}^{1}, s_{j} \in h_{S}^{2}}\left\{\max \left\{s_{i}, s_{j}\right\}\right\}$

$$
=\bigcup_{s_{j} \in h_{S}^{2}, s_{i} \in h_{S}^{h_{S}}}\left\{\max \left\{s_{j}, s_{i}\right\}\right\}=h_{S}^{2} \vee h_{S}^{1} ;
$$

(4) $h_{S}^{1} \wedge h_{S}^{2}=\bigcup_{s_{i} \in h_{S}^{1}, s_{j} \in h_{S}^{2}}\left\{\min \left\{s_{i}, s_{j}\right\}\right\}$

$$
=\bigcup_{s_{j} \in h_{S}^{2}, s_{i} \in h_{S}^{1}}\left\{\min \left\{s_{j}, s_{i}\right\}\right\}=h_{S}^{2} \wedge h_{S}^{1} .
$$

Theorem 4. (Associative) Let $h_{S}^{1}, h_{S}^{2}$ and $h_{S}^{3}$ be three EHFLTSS, then

(1) $h_{S}^{1} \oplus\left(h_{S}^{2} \oplus h_{S}^{3}\right)=\left(h_{S}^{1} \oplus h_{S}^{2}\right) \oplus h_{S}^{3}$;

(2) $h_{S}^{1} \otimes\left(h_{S}^{2} \otimes h_{S}^{3}\right)=\left(h_{S}^{1} \otimes h_{S}^{2}\right) \otimes h_{S}^{3}$;

(3) $h_{S}^{1} \vee\left(h_{S}^{2} \vee h_{S}^{3}\right)=\left(h_{S}^{1} \vee h_{S}^{2}\right) \vee h_{S}^{3}$;

(4) $h_{S}^{1} \wedge\left(h_{S}^{2} \wedge h_{S}^{3}\right)=\left(h_{S}^{1} \wedge h_{S}^{2}\right) \wedge h_{S}^{3}$.

\section{Proof.}


(1) $h_{S}^{1} \oplus\left(h_{S}^{2} \oplus h_{S}^{3}\right)=h_{S}^{1} \oplus\left(\bigcup_{s_{j} \in h_{S}^{2}, s_{k} \in h_{S}^{3}}\left\{s_{j+k}\right\}\right)$

$$
=\bigcup_{s_{i} \in h_{S}^{1}, s_{j} \in h_{S}^{2}, s_{k} \in h_{S}^{3}}\left\{s_{i} \oplus s_{j+k}\right\}=\bigcup_{s_{i} \in h_{S}^{1}, s_{j} \in h_{S}^{2}, s_{k} \in h_{S}^{3}}\left\{s_{i+j+k}\right\} \text {, }
$$$$
\left(h_{S}^{1} \oplus h_{S}^{2}\right) \oplus h_{S}^{3}=\left(\bigcup_{s_{i} \in h_{S}^{1}, s_{j} \in h_{S}^{2}}\left\{s_{i+j}\right\}\right) \oplus h_{S}^{3}
$$$$
=\bigcup_{s_{i} \in h_{S}^{1}, s_{j} \in h_{S}^{2}, s_{k} \in h_{S}^{3}}\left\{s_{i+j} \oplus s_{k}\right\}=\bigcup_{s_{i} \in h_{S}^{1}, s_{j} \in h_{S}^{2}, s_{k} \in h_{S}^{3}}\left\{s_{i+j+k}\right\} \text {; }
$$

(2) $h_{S}^{1} \otimes\left(h_{S}^{2} \otimes h_{S}^{3}\right)=h_{S}^{1} \otimes\left(\bigcup_{s_{j} \in h_{S}^{2}, s_{k} \in h_{S}^{3}}\left\{s_{j k}\right\}\right)$

$$
=\bigcup_{s_{i} \in h_{S}^{1}, s_{j} \in h_{S}^{2}, s_{k} \in h_{S}^{3}}\left\{s_{i} \otimes s_{j k}\right\}=\bigcup_{s_{i} \in h_{S}^{1}, s_{j} \in h_{S}^{2}, s_{k} \in h_{S}^{3}}\left\{s_{i j k}\right\},
$$$$
\left(h_{S}^{1} \otimes h_{S}^{2}\right) \otimes h_{S}^{3}=\left(\bigcup_{s_{i} \in h_{S}^{1}, s_{j} \in h_{S}^{2}}\left\{s_{i j}\right\}\right) \otimes h_{S}^{3}
$$$$
=\bigcup_{s_{i} \in h_{S}^{1}, s_{j} \in h_{S}^{2}, s_{k} \in h_{S}^{3}}\left\{s_{i j} \otimes s_{k}\right\}=\bigcup_{s_{i} \in h_{S}^{1}, s_{j} \in h_{S}^{2}, s_{k} \in h_{S}^{3}}\left\{s_{i j k}\right\} \text {; }
$$

(3) $h_{S}^{1} \vee\left(h_{S}^{2} \vee h_{S}^{3}\right)=h_{S}^{1} \vee\left(\bigcup_{s_{j} \in h_{S}^{2}, s_{k} \in h_{S}^{3}}\left\{\max \left\{s_{j}, s_{k}\right\}\right\}\right)$

$$
=\bigcup_{s_{i} \in h_{S}^{1}, s_{j} \in h_{S}^{2}, s_{k} \in h_{S}^{3}}\left\{\max \left\{s_{i}, \max \left\{s_{j}, s_{k}\right\}\right\}\right\}
$$$$
=\bigcup_{s_{i} \in h_{S}^{1}, s_{j} \in h_{S}^{2}, s_{k} \in h_{S}^{3}}\left\{\max \left\{s_{i}, s_{j}, s_{k}\right\}\right\} \text {, }
$$$$
\left(h_{S}^{1} \vee h_{S}^{2}\right) \vee h_{S}^{3}=\left(\bigcup_{s_{i} \in h_{S}^{1}, s_{j} \in h_{S}^{2}}\left\{\max \left\{s_{i}, s_{j}\right\}\right\}\right) \vee h_{S}^{3}
$$$$
=\bigcup_{s_{i} \in h_{S}^{1}, s_{j} \in h_{S}^{2}, s_{k} \in h_{S}^{3}}\left\{\max \left\{\max \left\{s_{i}, s_{j}\right\}, s_{k}\right\}\right\}
$$$$
=\bigcup_{s_{i} \in h_{S}^{1}, s_{j} \in h_{S}^{2}, s_{k} \in h_{S}^{3}}\left\{\max \left\{s_{i}, s_{j}, s_{k}\right\}\right\} \text {; }
$$

(4) $h_{S}^{1} \wedge\left(h_{S}^{2} \wedge h_{S}^{3}\right)=h_{S}^{1} \wedge\left(\bigcup_{s_{j} \in h_{S}^{2}, s_{k} \in h_{S}^{3}}\left\{\min \left\{s_{j}, s_{k}\right\}\right\}\right)$

$$
=\bigcup_{s_{i} \in h_{S}^{1}, s_{j} \in h_{S}^{2}, s_{k} \in h_{S}^{3}}\left\{\min \left\{s_{i}, \min \left\{s_{j}, s_{k}\right\}\right\}\right\}
$$$$
=\bigcup_{s_{i} \in h_{S}^{1}, s_{j} \in h_{S}^{2}, s_{k} \in h_{S}^{3}}\left\{\min \left\{s_{i}, s_{j}, s_{k}\right\}\right\} \text {, }
$$$$
\left(h_{S}^{1} \wedge h_{S}^{2}\right) \wedge h_{S}^{3}=\left(\bigcup_{s_{i} \in h_{S}^{1}, s_{j} \in h_{S}^{2}}\left\{\min \left\{s_{i}, s_{j}\right\}\right\}\right) \wedge h_{S}^{3}
$$$$
=\bigcup_{s_{i} \in h_{S}^{1}, s_{j} \in h_{S}^{2}, s_{k} \in h_{S}^{3}}\left\{\min \left\{\min \left\{s_{i}, s_{j}\right\}, s_{k}\right\}\right\}
$$$$
=\bigcup_{s_{i} \in h_{S}^{1}, s_{j} \in h_{S}^{2}, s_{k} \in h_{S}^{3}}\left\{\min \left\{s_{i}, s_{j}, s_{k}\right\}\right\} \text {. }
$$

Theorem 5. (Distributive) Let $h_{S}^{1}, h_{S}^{2}$ and $h_{S}$ be three EHFLTs, moreover, $\lambda \geq 0$, then we have:
(1) $\lambda\left(h_{S}^{1} \oplus h_{S}^{2}\right)=\lambda h_{S}^{1} \oplus \lambda h_{S}^{2}$;
(2) $\left(h_{S}^{1} \otimes h_{S}^{2}\right)^{\lambda}=\left(h_{S}^{1}\right)^{\lambda} \otimes\left(h_{S}^{2}\right)^{\lambda}$.

Proof. According to (3) and (4) of Definition 10, we have:

$$
\begin{aligned}
& s_{i} \in h_{S} \Leftrightarrow \lambda s_{i} \in \lambda h_{S}, s_{i} \in h_{S} \Leftrightarrow\left(s_{i}\right)^{\lambda} \in\left(h_{S}\right)^{\lambda} . \\
& \text { (1) } \lambda\left(h_{S}^{1} \oplus h_{S}^{2}\right)=\bigcup_{s_{i} \in h_{S}^{1}, s_{j} \in h_{S}^{2}}\left\{\lambda\left(s_{i} \oplus s_{j}\right)\right\}
\end{aligned}
$$

$$
\begin{gathered}
\quad=\bigcup_{s_{i} \in h_{S}^{1}, s_{j} \in h_{S}^{2}}\left\{\lambda s_{i} \oplus \lambda s_{j}\right\}, \\
\lambda h_{S}^{1} \oplus \lambda h_{S}^{2}=\bigcup_{\lambda s_{i} \in \lambda h_{S}^{1}, \lambda s_{j} \in \lambda h_{S}^{2}}\left\{\lambda s_{i} \oplus \lambda s_{j}\right\} \\
=\bigcup_{s_{i} \in h_{S}^{1}, s_{j} \in h_{S}^{2}}\left\{\lambda s_{i} \oplus \lambda s_{j}\right\} ; \\
\text { (2) }\left(h_{S}^{1} \otimes h_{S}^{2}\right)^{\lambda}=\bigcup_{s_{i} \in h_{S}^{1}, s_{j} \in h_{S}^{2}}\left\{\left(s_{i} \otimes s_{j}\right)^{\lambda}\right\} \\
=\bigcup_{s_{i} \in h_{S}^{1}, s_{j} \in h_{S}^{2}}\left\{\left(s_{i}\right)^{\lambda} \otimes\left(s_{j}\right)^{\lambda}\right\}, \\
\left(h_{S}^{1}\right)^{\lambda} \otimes\left(h_{S}^{2}\right)^{\lambda}=\bigcup_{\left(s_{i}\right)^{\lambda} \in\left(h_{S}^{1}\right)^{\lambda},\left(s_{j}\right)^{\lambda} \in\left(h_{S}^{2}\right)^{\lambda}\left\{\left(s_{i}\right)^{\lambda} \otimes\left(s_{j}\right)^{\lambda}\right\}} \\
=\bigcup_{s_{i} \in h_{S}^{1}, s_{j} \in h_{S}^{2}}\left\{\left(s_{i}\right)^{\lambda} \otimes\left(s_{j}\right)^{\lambda}\right\} .
\end{gathered}
$$

\subsection{Comparison laws of EHFLTs}

Rodriguez et al. ${ }^{19}$ utilized envelops of HFLTSs, interval linguistic terms, to compare information included in HFLTSs. However, as linguistic terms in EHFLTSs are not consecutive, envelops are not accurate for comparison. In this study, we introduce some concepts to distinguish two EHFLTs.

Definition 11. Given an EHFLT $h_{S}$,

$$
E\left(h_{S}\right)=\frac{1}{\# h_{S}}\left(\oplus_{s_{i} \in h_{S}} s_{i}\right)
$$

is called the expected linguistic term of $h_{S}$, where $\# h_{S}$ is the number of linguistic terms, $s_{i}$, in $h_{S}$.

Definition 12. Given an EHFLT $h_{S}, s_{i}$ and $s_{j}$ are the smallest and the biggest linguistic terms of $h_{S}$, then $D\left(h_{S}\right)=(j-i) /(2 t+1)$ is called the degree of hesitancy of $h_{S}$, where $2 t+1$ is the cardinality of linguistic term set $S$.

Expected linguistic term reflects the averaging linguistic term of an EHFLT, and degree of hesitancy represents the degree of uncertainty in evaluation. If the smallest and the biggest linguistic terms of $h_{S}$ are original linguistic terms in $S$, then $D\left(h_{S}\right) \in[0,1]$. However, if at least one of the two comes from $\bar{S}$, the boundary of $D\left(h_{S}\right)$ does not hold any more. Using these definitions, we can compare two EHFLTs by the following method.

Definition 13. Given two EHFLTs $h_{S}^{1}$ and $h_{S}^{2}$, then

(1) if $E\left(h_{S}^{1}\right)<E\left(h_{S}^{2}\right)$, then $h_{S}^{1}$ is smaller than $h_{S}^{2}$, denoted by $h_{S}^{1}<h_{S}^{2}$; 
(2) if $E\left(h_{S}^{1}\right)=E\left(h_{S}^{2}\right)$, then

a) if $D\left(h_{S}^{1}\right)>D\left(h_{S}^{2}\right)$, then $h_{S}^{1}$ is smaller than $h_{S}^{2}$, denoted by $h_{S}^{1}<h_{S}^{2}$;

b) if $D\left(h_{S}^{1}\right)=D\left(h_{S}^{2}\right)$, then $h_{S}^{1}$ and $h_{S}^{2}$ represent the same information, denoted by $h_{S}^{1} \cong h_{S}^{2}$.

Example 5. Let $S$ be the linguistic term set in Example 2. $h_{S}^{1}=\left\{s_{0}\right\}, h_{S}^{2}=\left\{s_{-1}, s_{1}\right\}, h_{S}^{3}=\left\{s_{0}, s_{1}, s_{3}\right\}$, then:

$$
E\left(h_{S}^{1}\right)=s_{0}, E\left(h_{S}^{2}\right)=s_{0}, E\left(h_{S}^{3}\right)=s_{1.333} \text {, }
$$$$
D\left(h_{S}^{1}\right)=0, D\left(h_{S}^{2}\right)=2 / 7, D\left(h_{S}^{3}\right)=3 / 7 \text {, }
$$

then we have $E\left(h_{S}^{1}\right)=E\left(h_{S}^{2}\right)<E\left(h_{S}^{3}\right)$, $D\left(h_{S}^{1}\right)<D\left(h_{S}^{2}\right)$. Therefore, $h_{S}^{2}<h_{S}^{1}<h_{S}^{3}$.

Note that, for two EHFLTs, $h_{s}^{1}$ and $h_{s}^{2}$, if $h_{s}^{1}=h_{S}^{2}$, we mean the elements in $h_{S}^{1}$ and $h_{S}^{2}$ are absolutely the same; if $h_{S}^{1} \cong h_{S}^{2}$, we mean the expected linguistic terms and the degrees of hesitancy of the two are equal, as in Definition 13.

\section{Some aggregation operators}

We may need to aggregate a set of EHFLTs to obtain overall satisfaction degree of an alternative before final decision is made. Thus we need to develop a function or mechanism to aggregate evaluations taking the form of EHFLTs. Motivated by Torra ${ }^{8}$, we propose an extension principle to export operators on linguistic term sets to EHFLTs in this section. Based on which, we present some specific aggregation operators for potential application. Some properties of aggregation operators are also discussed. The extension principle is defined first.

Definition 14. Let $\Theta$ be a function $\Theta: \bar{S}^{n} \rightarrow \bar{S}$, where $\bar{S}=\left\{s_{\alpha} \mid \alpha \in[-q, q]\right\}, H=\left\{h_{S}^{1}, h_{S}^{2}, \cdots, h_{S}^{n}\right\}$ be a set of EHFLTS on the reference set $X$. Then the extension of $\Theta$ on $H$ is defined for each $x$ in $X$ by:

$\Theta_{H}(x)=\bigcup_{\left(s_{\alpha_{1}}, s_{\alpha_{2}}, \cdots, s_{\alpha_{n}}\right) \in\left\{h_{s}^{1} \times h_{S}^{2} \times \cdots \times h_{s}^{n}\right\}}\left\{\Theta\left(s_{\alpha_{1}}, s_{\alpha_{2}}, \cdots, s_{\alpha_{n}}\right)\right\}$.

Similar to (7), in the rest of this section, two sets of EHFLTs are denoted by $H=\left\{h_{S}^{j}\right\}$ and $\dot{H}=\left\{\dot{h}_{S}^{j}\right\}$, $j=1,2, \cdots, n$. The number of linguistic terms in $h_{S}^{j}$ and $\dot{h}_{S}^{j}$ are denoted by $\# h_{S}^{j}$ and $\# \dot{h_{S}^{j}}$. Linguistic terms in $h_{s}^{j}$ and $\dot{h}_{S}^{j}$ are denoted by $s_{\alpha_{j}}$ and $s_{\dot{\alpha}_{j}}$ respectively. Furthermore, the $i_{j}$ th linguistic terms in $h_{S}^{j}$ and $\dot{h}_{S}^{j}$ are specified by $s_{\alpha_{j_{j} j_{j}}}$ and $s_{\dot{\alpha}_{j_{i j}}}$ sometimes. We shall define two classes of aggregation operators according to the form of weighting vector in the next subsections.

\subsection{Aggregation operators with linguistic weights}

First, we present some aggregation operators when weights of EHFLTs are linguistic terms. Given a set of EHFLTs are denoted by $\left\{h_{S}^{j}\right\}, j=1,2, \cdots, n$, suppose that their corresponding weights take the form of linguistic terms from $S$. Herrera and Herrera-Viedma ${ }^{36}$ provided the linguistic weighted disjunction (LWD) operators in the setting values and weights of objects are represented by simple linguistic terms. As the extension of the LWD operator, we define the following operator using Definition 14.

Definition 15. Let $\left\{h_{S}^{j}\right\}, j=1,2, \cdots, n$ be a set of EHFLTS, $\omega=\left(\omega_{1}, \cdots, \omega_{n}\right)$ be the weighting vector of $\left\{h_{S}^{j}\right\}, \omega_{j} \in S$ for all $j$. A mapping EHFLWD: $S^{n} \rightarrow S$ is called an extended hesitant fuzzy linguistic weighted disjunction (EHFLWD) operator of dimension $n$ if

$$
\begin{aligned}
\text { EHFLWD }\left(h_{S}^{1}, h_{S}^{2}, \cdots, h_{S}^{n}\right)=\underset{j=1}{\stackrel{n}{\vee}}\left(\omega_{j} \wedge h_{S}^{j}\right) \\
=\underset{j=1}{\vee}\left(\bigcup_{s_{\alpha_{j}} \in h_{S}^{j}} \min \left\{\omega_{j}, s_{\alpha_{j}}\right\}\right) \\
=\bigcup_{s_{\alpha_{1}} \in h_{S}^{1}, \cdots, s_{\alpha_{n}} \in \epsilon_{S}^{n}}\left\{\max _{j}\left\{\min \left\{\omega_{j}, s_{\alpha_{j}}\right\}\right\} .\right.
\end{aligned}
$$

If $\omega=\left(s_{t}, \cdots, s_{t}\right)$, where $s_{t}$ is the largest linguistic term in (1), then $\omega_{j} \wedge h_{S}^{j}=h_{S}^{j}$, therefore,

$$
\operatorname{EHFLWD}\left(h_{S}^{1}, h_{S}^{2}, \cdots, h_{S}^{n}\right)={\underset{j=1}{n}}_{\vee} h_{S}^{j} .
$$

However, $\underset{j=1}{\vee} h_{S}^{j} \neq \max _{j}\left\{h_{S}^{j}\right\}$ in general.

The ordered weighted averaging (OWA) operator ${ }^{37}$ provides an aggregation strategy to lie between the max and min operators because of its re-ordering step. In linguistic setting, Yager ${ }^{38}$ presented an ordinal form of the OWA operator. Motivated by which, we extend the OWA operator to the extended hesitant fuzzy linguistic setting.

Definition 16. Let $\left\{h_{S}^{j}\right\}, j=1,2, \cdots, n$ be a set of EHFLTS. An extended hesitant fuzzy ordinal OWA (EHFOOWA) operator of dimension $n$ is a mapping EHFOOWA: $S^{n} \rightarrow S$, which has associated with a 
linguistic weighting vector $w=\left(w_{1}, \cdots, w_{n}\right)$ with $w_{j} \in S$ for all $j$ such that

$$
\begin{aligned}
& \operatorname{EHFOOWA}\left(h_{S}^{1}, h_{S}^{2}, \cdots, h_{S}^{n}\right)=\underset{j=1}{\vee}\left(w_{j} \wedge h_{S}^{\sigma(j)}\right) \\
& ={\underset{j=1}{V}}^{n}\left(\bigcup_{s_{\alpha_{\sigma(j)}} \in \sigma_{S}^{\sigma(j)}} \min \left\{w_{j}, s_{\alpha_{\sigma(j)}}\right\}\right) \\
& =\bigcup_{s_{\alpha_{\sigma(1)}} \in h_{S}^{\sigma(1)}, \cdots, s_{\alpha_{\sigma(n)}} \in h_{S}^{\sigma(n)}}\left\{\max _{j}\left\{\min \left\{w_{j}, s_{\alpha_{\sigma(j)}}\right\}\right\}\right\} \text {, }
\end{aligned}
$$

where $h_{S}^{\sigma(j)}$ is the $j$ th largest of the $h_{S}^{j}$.

If $w=\left(s_{t}, s_{-t}, \cdots, s_{-t}\right)$, then $w_{1} \wedge h_{S}^{\sigma(1)}=h_{S}^{\sigma(1)}$ and $w_{j} \wedge h_{S}^{\sigma(j)}=w_{j}$ for $j=2, \cdots, n$, then

$$
\begin{aligned}
\operatorname{EHFOOWA}\left(h_{S}^{1}, h_{S}^{2}, \cdots, h_{S}^{n}\right) & =h_{S}^{\sigma(1)} \vee s_{-t} \vee \cdots \vee s_{-t} \\
& =h_{S}^{\sigma(1)}=\max _{j}\left\{h_{S}^{j}\right\} .
\end{aligned}
$$

The EHFOOWA operator is reduced to the extended hesitant fuzzy linguistic $\max \left(\mathrm{EHFLM}_{1}\right)$ operator. Similarly, if $w=\left(s_{-t}, \cdots, s_{-t}, s_{t}\right)$,

$$
\operatorname{EHFOOWA}\left(h_{S}^{1}, h_{S}^{2}, \cdots, h_{S}^{n}\right)=\min _{j}\left\{h_{S}^{j}\right\} .
$$

The EHFOOWA operator is reduced to the extended hesitant fuzzy linguistic min (EHFLM2) operator in this case.

Consider that the LWD operator weights only the values themselves, while the ordinal OWA operator weights the re-ordered positions of the values only, $\mathrm{Xu}$ 39 proposed an ordinal hybrid aggregation (OHA) operator to reflect the importance degrees of both the linguistic arguments and their ordered positions. Based on the same idea, we develop the follow aggregation operator.

Definition 17. Let $\left\{h_{S}^{j}\right\}, j=1,2, \cdots, n$ be a set of EHFLTs. An extended hesitant fuzzy ordinal hybrid aggregation (EHFOHA) operator of dimension $n$ is a mapping EHFOHA: $S^{n} \rightarrow S$, which has associated with a linguistic weighting vector $w=\left(w_{1}, \cdots, w_{n}\right)$ with $w_{j} \in S$ for all $j$ such that

$$
\begin{aligned}
& \operatorname{EHFOHA}\left(h_{S}^{1}, h_{S}^{2}, \cdots, h_{S}^{n}\right)={\underset{j=1}{\vee}}^{n}\left(w_{j} \wedge h_{S}^{\dot{\sigma(j)}}\right) \\
& ={\underset{j=1}{\vee}}^{n}\left(\bigcup_{s_{\alpha_{\delta(j)}} \in h_{S}^{\dot{\sigma}(j)}} \min \left\{w_{j}, s_{\alpha_{\sigma(j)}}\right\}\right) \\
& =\bigcup_{s_{\alpha_{\delta(1)}} \in h_{S}^{\dot{\sigma}(1)}, \cdots, s_{\alpha_{\delta(n)}} \in h_{S}^{\dot{\sigma}(n)}}\left\{\max _{j}\left\{\min \left\{w_{j}, s_{\alpha_{\delta(j)}}\right\}\right\}\right\},
\end{aligned}
$$

where $h_{S}^{\sigma(j)}$ is the $j$ th largest of the linguistic weighted arguments $\bar{h}_{S}^{j}=\omega_{j} \wedge h_{S}^{j}, \omega=\left(\omega_{1}, \cdots, \omega_{n}\right)$ be the weighting vector of $\left\{h_{S}^{j}\right\}, \omega_{j} \in S$ for all $j$.

Especially, if $w=\left(s_{t}, s_{t} \cdots, s_{t}\right)$, then $w_{j} \wedge h_{S}^{\dot{\sigma}(j)}$ $=h_{S}^{\dot{\sigma}(j)}$ holds for any $h_{S}^{\dot{\sigma}(j)}$,

$$
\begin{gathered}
\operatorname{EHFOHA}\left(h_{S}^{1}, h_{S}^{2}, \cdots, h_{S}^{n}\right)={ }_{j=1}^{n}\left(w_{j} \wedge h_{S}^{\dot{\sigma}(j)}\right) \\
={ }_{j=1}^{n} h_{S}^{\dot{\sigma(j)}}={ }_{j=1}^{n}\left(\omega_{j} \wedge h_{S}^{j}\right)
\end{gathered}
$$

thus the EHFOHA operator is reduced to the EHFLWD operator. If $\omega=\left(s_{t}, s_{t} \cdots, s_{t}\right)$, then for any $h_{S}^{j}$, $\omega_{j} \wedge h_{S}^{j}=h_{S}^{j}$ holds, then

$$
\begin{gathered}
\operatorname{EHFOHA}\left(h_{S}^{1}, h_{S}^{2}, \cdots, h_{S}^{n}\right)=\vee_{j=1}^{n}\left(w_{j} \wedge h_{S}^{\dot{\sigma(j)}}\right) \\
={ }_{j=1}^{n}\left(w_{j} \wedge h_{S}^{\sigma(j)}\right),
\end{gathered}
$$

thus the EHFOHA operator is reduced to the EHFOOWA operator.

\subsection{Aggregation operators with numerical weights}

Except for linguistic weights, numerical weights are often used in application as well. In this case, several aggregation operators are developed in linguistic setting, such as the linguistic weighted averaging (LWA) operator ${ }^{40}$, the linguistic OWA operator ${ }^{40}$, the linguistic hybrid aggregation (LHA) operator ${ }^{41}$, the induced linguistic OWA operator ${ }^{40}$ and so on. Based on the extension principle and some existing linguistic aggregation operator, we define some new aggregation operators as follows.

Definition 18. Let $\left\{h_{S}^{j}\right\}, j=1,2, \cdots, n$ be a set of EHFLTs, $\omega=\left(\omega_{1}, \cdots, \omega_{n}\right)$ be the weighting vector of $\left\{h_{S}^{j}\right\}$ such that $\sum \omega_{j}=1, \omega_{j} \in[0,1]$ for all $j$. A mapping EHFLW'Al: $\bar{S}^{n} \rightarrow \bar{S}$ is called an extended hesitant fuzzy linguistic weighted averaging (EHFLWA) operator of dimension $n$ if

$$
\begin{aligned}
\text { EHFLWA } & W\left(h_{S}^{1}, h_{S}^{2}, \cdots, h_{S}^{n}\right)=\bigoplus_{j=1}^{n}\left(\omega_{j} h_{S}^{j}\right) \\
= & \bigoplus_{j=1}^{n}\left(\bigcup_{s_{\alpha_{j}} \in h_{S}^{j}}\left\{\omega_{j} s_{\alpha_{j}}\right\}\right) \\
= & \bigcup_{s_{\alpha_{1}} \in h_{S}^{1}, \cdots, s_{\alpha_{n}} \in h_{S}^{n}}\left\{\omega_{1} s_{\alpha_{1}} \oplus \cdots \oplus \omega_{n} s_{\alpha_{n}}\right\}
\end{aligned}
$$




$$
=\bigcup_{s_{\alpha_{1}} \in h_{S}^{1}, \cdots, s_{\alpha_{n}} \in h_{S}^{n}}\left\{s_{\dot{\alpha}}\right\},
$$

where $\dot{\bar{\alpha}}=\sum_{j=1}^{n} \omega_{j} \alpha_{j}$.

The EHFLWA operator extend both the weighted averaging (WA) operator ${ }^{42}$ and the LWA operator. Especially, if $\omega=(1 / n, 1 / n, \cdots, 1 / n)$, the EHFLWA operator is reduced to the extended hesitant fuzzy linguistic averaging (EHFLA) operator:

$$
\begin{aligned}
& \operatorname{EHFLWA}\left(h_{S}^{1}, h_{S}^{2}, \cdots, h_{S}^{n}\right) \\
& \quad=\bigcup_{s_{\alpha_{1}} \in h_{S}^{1}, \cdots, s_{\alpha_{n}} \in h_{S}^{n}}\left\{1 / n\left(s_{\alpha_{1}} \oplus \cdots \oplus s_{\alpha_{n}}\right)\right\} .
\end{aligned}
$$

The fundamental aspect of the LWA2 operator is that it takes into account the importance of each EHFLT. By extending the OWA operator and the LOWA operator, we define the extended hesitant fuzzy linguistic OWA (EHFLOWA) operator in the following definition.

Definition 19. Let $\left\{h_{S}^{j}\right\}, j=1,2, \cdots, n$ be a set of EHFLTS. An EHFLOWA operator of dimension $n$ is a mapping EHFOOWA: $\bar{S}^{n} \rightarrow \bar{S}$, which has associated with a linguistic weighting vector $w=\left(w_{1}, \cdots, w_{n}\right)$ with $\sum_{j=1}^{n} w_{j}=1, w_{j} \in[0,1]$ for all $j$, such that

$$
\begin{aligned}
& \operatorname{EHFLOWA}\left(h_{S}^{1}, h_{S}^{2}, \cdots, h_{S}^{n}\right)=\bigoplus_{j=1}^{n}\left(w_{j} h_{S}^{\sigma(j)}\right) \\
& =\bigoplus_{j=1}^{n}\left(\bigcup_{s_{\beta_{j}} \in h_{S}^{\sigma(j)}}\left\{w_{j} s_{\beta_{j}}\right\}\right) \\
& =\bigcup_{s_{\beta_{1}} \in h_{S}^{\sigma(1)}, \cdots, s_{\beta_{n}} \in h_{S}^{\sigma(n)}}\left\{w_{1} s_{\beta_{1}} \oplus \cdots \oplus w_{n} s_{\beta_{n}}\right\} \\
& =\bigcup_{s_{\beta_{1}} \in h_{S}^{\sigma(1)}, \cdots, s_{\beta_{n}} \in h_{S}^{\sigma(n)}}\left\{s_{\bar{\beta}}\right\} \text {, }
\end{aligned}
$$

where $\bar{\beta}=\sum_{j=1}^{n} w_{j} \beta_{j}, h_{S}^{\sigma(j)}$ is the $j$ th largest of the $h_{S}^{j}$.

Especially if $w=(1,0, \cdots, 0)$, then $w_{1} h_{S}^{\sigma(1)}=h_{S}^{\sigma(1)}$ and $w_{j} h_{S}^{\sigma(j)}=s_{0}$ for $j=2, \cdots, n$, then

$$
\begin{aligned}
& \text { EHFLOWA }\left(h_{S}^{1}, h_{S}^{2}, \cdots, h_{S}^{n}\right) \\
& \quad=h_{S}^{\sigma(1)} \oplus s_{0} \oplus \cdots \oplus s_{0}=h_{S}^{\sigma(1)}=\max _{j}\left\{h_{S}^{j}\right\} .
\end{aligned}
$$

The EHFLOWA operator is reduced to the EHFLM $_{1}$ operator. Similarly, if $w=(0, \cdots, 0,1)$,

$$
\operatorname{EHFLOWA}\left(h_{S}^{1}, h_{S}^{2}, \cdots, h_{S}^{n}\right)=\min \left\{h_{S}^{j}\right\} .
$$

The EHFLOWA operator is reduced to the EHFLM $_{2}$ operator.

The fundamental aspect of the EHFLOWA operator is that it reorders the input arguments based on their values. From Definitions 18 and 19, it is clear that the EHFLWA operator weights the input EHFLTs, while the EHFLOWA operator weights the ordered position instead. The weights represent distinct aspects of inputs in these two operators. We present the following operator to overcome this drawback.

Definition 20. Let $\left\{h_{S}^{j}\right\}, j=1,2, \cdots, n$ be a set of EHFLTs. An extended hesitant fuzzy linguistic hybrid aggregation (EHFLHA) operator of dimension $n$ is a mapping EHFLHA: $\bar{S}^{n} \rightarrow \bar{S}$, which has associated with a linguistic weighting vector $w=\left(w_{1}, \cdots, w_{n}\right)$ with $\sum_{j=1}^{n} w_{j}=1, w_{j} \in[0,1]$ for all $j$, such that

$$
\begin{aligned}
\operatorname{EHFLHA} & \left(h_{S}^{1}, h_{S}^{2}, \cdots, h_{S}^{n}\right)=\bigoplus_{j=1}^{n}\left(w_{j} h_{S}^{\sigma(j)}\right) \\
= & \bigoplus_{j=1}^{n}\left(\bigcup_{s_{\beta_{j}} \in h_{S}^{\sigma(j)}}\left\{w_{j} s_{\dot{\beta}_{j}}\right\}\right) \\
= & \bigcup_{s_{\beta_{1}} \in h_{S}^{\delta(1)}, \cdots, s_{\beta_{n}} \in h_{S}^{\delta(n)}}\left\{w_{1} s_{\dot{\beta}_{1}} \oplus \cdots \oplus w_{n} s_{\dot{\beta}_{n}}\right\} \\
= & \bigcup_{s_{\beta_{1}} \in h_{S}^{\sigma(1)}, \cdots, s_{\beta_{n}} \in h_{S}^{\sigma(n)}}\left\{s_{\dot{\beta}}\right\},
\end{aligned}
$$

where $\dot{\bar{\beta}}=\sum_{j=1}^{n} w_{j} \dot{\beta}_{j}, h_{S}^{\dot{\sigma}(j)}$ is the $j$ th largest of the linguistic weighted arguments $\bar{h}_{S}^{j}=n \omega_{j} h_{S}^{j}$, $\omega=\left(\omega_{1}, \cdots, \omega_{n}\right)$ be the weighting vector of $\left\{h_{S}^{j}\right\}$ such that $\sum_{j=1}^{n} \omega_{j}=1, \omega_{j} \in[0,1]$ for all $j$, and $n$ is the balancing coefficient.

Especially, if $w=(1 / n, 1 / n, \cdots, 1 / n)$, according to (1) of Theorem 3 and (1) of Theorem 4,

$$
\begin{aligned}
\operatorname{EHFLHA}\left(h_{S}^{1}, h_{S}^{2}, \cdots, h_{S}^{n}\right)=\bigoplus_{j=1}^{n}\left(\frac{1}{n} h_{S}^{\dot{\sigma}(j)}\right) \\
=\bigoplus_{j=1}^{n}\left(\frac{1}{n} n \omega_{j} h_{S}^{j}\right)=\bigoplus_{j=1}^{n}\left(\omega_{j} h_{S}^{j}\right),
\end{aligned}
$$

then the EHFLHA operator is reduced to the EHFLWA operator. If $\omega=(1 / n, 1 / n, \cdots, 1 / n)$, then $\bar{h}_{S}^{j}=h_{S}^{j}$, we have

$$
\operatorname{EHFLHA}\left(h_{S}^{1}, h_{S}^{2}, \cdots, h_{S}^{n}\right)=\bigoplus_{j=1}^{n}\left(w_{j} h_{S}^{\dot{\sigma(j)}}\right)=\bigoplus_{j=1}^{n}\left(w_{j} h_{S}^{\sigma(j)}\right),
$$

then the EHFLHA operator is reduced to the EHFLOWA operator. It is clear that the LHA operator generalizes both the EHFLWA and EHFLOWA operators, and reflects the importance degrees of both the input arguments and their ordered positions. 


\subsection{Properties}

We will discuss some properties of the presented aggregation operators in this subsection. Because of the operation "U", most of the operators do not possess excellent mathematical properties, such as monotonicity, idempotency, commutativity and boundary. But, luckily, we will see some operators own properties like these four.

Theorem 6. Let $\left\{h_{S}^{j}\right\}$ and $\left\{\dot{h}_{S}^{j}\right\}, j=1,2, \cdots, n$, be two sets of EHFLTs. If $\exists i \in\{1, \cdots, n\}$ satisfies $\# h_{S}^{i}=\# \dot{h}_{S}^{i}=N_{i}$, and $\forall s_{\alpha_{i j_{i}}} \in h_{S}^{i}, s_{\dot{\alpha}_{i j i}} \in \dot{h}_{S}^{i}, s_{\alpha_{i_{j_{i}}}} \leq s_{\dot{\alpha}_{\dot{\alpha}_{i j}}}$ holds. For $j=1, \cdots, n, j \neq i, h_{S}^{i}=\dot{h}_{S}^{i}$. Then

$$
\operatorname{EHFLWD}\left(h_{S}^{1}, h_{S}^{2}, \cdots, h_{S}^{n}\right) \leq \operatorname{EHFLWD}\left(\dot{h}_{S}^{1}, \dot{h}_{S}^{2}, \cdots, \dot{h}_{S}^{n}\right) .
$$

Proof. Since $s_{\alpha_{i_{i j}}} \leq s_{\dot{\alpha}_{i_{j_{j}}}}$, then $\omega_{i} \wedge s_{\alpha_{i_{i j}}} \leq \omega_{i} \wedge s_{\dot{i}_{i_{j}}}$ for any $\omega_{i} \in S$. Then

$$
\bigcup_{s_{\alpha_{i_{j i}}} \in h_{S}^{i}}\left\{\omega_{i} \wedge s_{\alpha_{i_{i j}}}\right\} \leq \bigcup_{s_{\dot{\alpha}_{i_{j}}} \in h_{S}^{i}}\left\{\omega_{i} \wedge s_{\dot{\alpha}_{i_{j_{i}}}}\right\}
$$

which means $\omega_{i} \wedge h_{S}^{i} \leq \omega_{i} \wedge \dot{h}_{S}^{i}$. Thus

$$
\begin{array}{rl}
E H F L & W D\left(h_{S}^{1}, h_{S}^{2}, \cdots, h_{S}^{n}\right) \\
& =\left(\omega_{1} \wedge h_{S}^{1}\right) \vee \cdots \vee\left(\omega_{i} \wedge h_{S}^{i}\right) \vee \cdots \vee\left(\omega_{n} \wedge h_{S}^{n}\right) \\
& \leq\left(\omega_{1} \wedge h_{S}^{1}\right) \vee \cdots \vee\left(\omega_{i} \wedge \dot{h}_{S}^{i}\right) \vee \cdots \vee\left(\omega_{n} \wedge h_{S}^{n}\right) \\
& =\left(\omega_{1} \wedge \dot{h}_{S}^{1}\right) \vee \cdots \vee\left(\omega_{i} \wedge \dot{h}_{S}^{i}\right) \vee \cdots \vee\left(\omega_{n} \wedge \dot{h}_{S}^{n}\right) \\
& =\text { EHFLWD }\left(\dot{h}_{S}^{1}, \dot{h}_{S}^{2}, \cdots, \dot{h}_{S}^{n}\right) .
\end{array}
$$

Theorem 7. (Quasi-Boundary) Let $\left\{h_{S}^{j}\right\}, j=1,2, \cdots, n$, be a set of EHFLTs. Then

$$
s_{-L} \leq \operatorname{EHFLWD}\left(h_{S}^{1}, h_{S}^{2}, \cdots, h_{S}^{n}\right) \leq s_{L},
$$

where $s_{-L}=\min _{j}\left\{\min \left\{\omega_{j}, \min _{s_{\alpha_{j}} \in h_{S}^{j}}\left\{s_{\alpha_{j}}\right\}\right\}\right\}$,

$$
s_{L}=\max _{j}\left\{\max \left\{\omega_{j}, \max _{s_{\alpha_{j}} \in h_{s}^{j}}\left\{s_{\alpha_{j}}\right\}\right\}\right\} \text {. }
$$

Proof. For any $s_{\alpha_{1}} \in h_{S}^{1}, \cdots, s_{\alpha_{n}} \in h_{S}^{n}$, we have

$$
\begin{aligned}
\min _{j}\{\min & \left.\left\{\omega_{j}, \min _{s_{\alpha_{j}} \in h_{S}^{j}}\left\{s_{\alpha_{j}}\right\}\right\}\right\} \leq \max _{j}\left\{\min \left\{\omega_{j}, s_{\alpha_{j}}\right\}\right\} \\
& \leq \max _{j}\left\{\max \left\{\omega_{j}, \max _{s_{\alpha_{j}} \in h_{S}^{j}}\left\{s_{\alpha_{j}}\right\}\right\}\right\} .
\end{aligned}
$$

Then

$$
s_{-L} \leq E\left(\bigcup_{s_{\alpha_{1}} \in h_{S}^{1}, \cdots, s_{\alpha_{n}} \in h_{S}^{n}}\left\{\max _{j}\left\{\min \left\{\omega_{j}, s_{\alpha_{j}}\right\}\right\}\right\}\right) \leq s_{L} .
$$

Thus $s_{-L} \leq \operatorname{EHFLWD}\left(h_{S}^{1}, h_{S}^{2}, \cdots, h_{S}^{n}\right) \leq s_{L}$.
Similarly, using the same approach, we can easily proof the following theorem.

Theorem 8. (Quasi-Boundary) Let $\left\{h_{S}^{j}\right\}, j=1,2, \cdots, n$, be a set of EHFLTs. Then

$$
s_{-L} \leq \text { EHFOOWA }\left(h_{S}^{1}, h_{S}^{2}, \cdots, h_{S}^{n}\right) \leq s_{L},
$$

where $s_{-L}=\min _{j}\left\{\min \left\{w_{j}, \min _{s_{\alpha_{j}} \in h_{S}^{j}}\left\{s_{\alpha_{j}}\right\}\right\}\right\}$,

$s_{L}=\max _{j}\left\{\max \left\{w_{j}, \max _{s_{\alpha_{j}} \in h_{S}^{j}}\left\{s_{\alpha_{j}}\right\}\right\}\right\}$.

Theorem 9. (Commutativity) Let $\left\{h_{S}^{j}\right\}, j=1,2, \cdots, n$, be a set of EHFLTs. Then

(1) $\operatorname{EHFOOWA}\left(h_{S}^{1}, h_{S}^{2}, \cdots, h_{S}^{n}\right)$

$$
=\operatorname{EHFOOWA}\left(\dot{h}_{S}^{1}, \dot{h}_{S}^{2}, \cdots, \dot{h}_{S}^{n}\right),
$$

(2) $\operatorname{EHFLOWA}\left(h_{S}^{1}, h_{S}^{2}, \cdots, h_{S}^{n}\right)$

$$
=\operatorname{EHFLOWA}\left(\dot{h}_{S}^{1}, \dot{h}_{S}^{2}, \cdots, \dot{h}_{S}^{n}\right) \text {, }
$$

where $\dot{h}_{S}^{1}, \dot{h}_{S}^{2}, \cdots, \dot{h}_{S}^{n}$ is any permutation of $h_{S}^{1}, h_{S}^{2}, \cdots, h_{S}^{n}$.

Proof. Since $\dot{h}_{S}^{1}, \dot{h}_{S}^{2}, \cdots, \dot{h}_{S}^{n}$ is any permutation of $h_{S}^{1}, h_{S}^{2}, \cdots, h_{S}^{n}$, then $h_{S}^{\sigma(j)}=\dot{h}_{S}^{\sigma(j)}$ for all $j$.

(1) $\operatorname{EHFOOWA}\left(h_{S}^{1}, h_{S}^{2}, \cdots, h_{S}^{n}\right)$

$$
\begin{aligned}
& =\vee_{j=1}^{n}\left(w_{j} \wedge h_{S}^{\sigma(j)}\right)=\vee_{j=1}^{n}\left(w_{j} \wedge \dot{h}_{S}^{\sigma(j)}\right) \\
& =\operatorname{EHFOOWA}\left(\dot{h}_{S}^{1}, \dot{h}_{S}^{2}, \cdots, \dot{h}_{S}^{n}\right) ;
\end{aligned}
$$

(2) $\operatorname{EHFLOWA}\left(h_{S}^{1}, h_{S}^{2}, \cdots, h_{S}^{n}\right)$

$$
\begin{aligned}
& =\bigoplus_{j=1}^{n}\left(w_{j} h_{S}^{\sigma(j)}\right)=\bigoplus_{j=1}^{n}\left(w_{j} \dot{h}_{S}^{\sigma(j)}\right) \\
& =\operatorname{EHFLOWA}\left(\dot{h}_{S}^{1}, \dot{h}_{S}^{2}, \cdots, \dot{h}_{S}^{n}\right) .
\end{aligned}
$$

Theorem 10. (Quasi-Idempotency) Let $h_{S}$ be an EHFLT. Then

$$
\operatorname{EHFLWA}\left(h_{S}, h_{S}, \cdots, h_{S}\right) \cong h_{S} .
$$

Proof. Suppose $\# h_{S}=N, h_{S}=\left\{s_{\beta_{1}}, s_{\beta_{2}}, \cdots, s_{\beta_{N}}\right\}$, then

$$
E\left(h_{S}\right)=\frac{1}{N} s_{\beta_{1}+\cdots+\beta_{N}}=s_{\frac{1}{N} \sum_{j=1}^{N} \beta_{j}} .
$$

First, we prove

$$
E\left(\operatorname{EHFLWA}\left(h_{S}, h_{S}, \cdots, h_{S}\right)\right)=E\left(h_{S}\right)
$$

by using mathematical induction on $n$.

(1) When $n=2$,

$\operatorname{EHFLWA}\left(h_{S}, h_{S}\right)=\omega_{1} h_{S} \oplus \omega_{2} h_{S}$ 


$$
=\bigcup_{s_{\alpha_{1}} \in h_{S}, s_{\alpha_{2}} \in h_{S}}\left\{s_{\omega_{1} \alpha_{1}+\omega_{2} \alpha_{2}}\right\} .
$$

For all the possible values of $\alpha_{1}$ and $\alpha_{2}$, the derived indices of virtual linguistic term $s$ are listed as follows. (All the possible values of $\alpha_{1}$ are listed in the first column, while all the possible values of $\alpha_{2}$ are listed in the first row.)

$$
\left.\begin{array}{ccccc} 
& \beta_{1} & \beta_{2} & \cdots & \beta_{N} \\
\beta_{1} & \omega_{1} \beta_{1}+\omega_{2} \beta_{1} & \omega_{1} \beta_{1}+\omega_{2} \beta_{2} & \cdots & \omega_{1} \beta_{1}+\omega_{2} \beta_{N} \\
\beta_{2} & \omega_{1} \beta_{2}+\omega_{2} \beta_{1} & \omega_{1} \beta_{2}+\omega_{2} \beta_{2} & \cdots & \omega_{1} \beta_{2}+\omega_{2} \beta_{N} \\
\vdots & \vdots & \vdots & \ddots & \vdots \\
\beta_{N} & \omega_{1} \beta_{N}+\omega_{2} \beta_{1} & \omega_{1} \beta_{N}+\omega_{2} \beta_{2} & \cdots & \omega_{1} \beta_{N}+\omega_{2} \beta_{N}
\end{array}\right)
$$

The sum of the indices in the bracket is:

$$
N \omega_{1} \sum_{j=1}^{N} \beta_{j}+N \omega_{2} \sum_{j=1}^{N} \beta_{j}=N \sum_{j=1}^{N} \beta_{j} .
$$

Thus

$$
E\left(\operatorname{EHFLWA}\left(h_{S}, h_{S}\right)\right)=s_{\frac{1}{N^{2}} N \sum_{j=1}^{N} \beta_{j}}=s_{\frac{1}{N} \sum_{j=1}^{N} \beta_{j}}=E\left(h_{S}\right) .
$$

(2) If $E\left(E H F L W A\left(h_{S}, h_{S}, \cdots, h_{S}\right)\right)=E\left(h_{S}\right)$ hold for $n=k$, that is

$$
E\left(\omega_{1} h_{S} \oplus \cdots \oplus \omega_{k} h_{S}\right)=E\left(h_{S}\right), \text { where } \sum_{j=1}^{k} \omega_{j}=1 .
$$

Then when $n=k+1$, according to some trivial computing similar to the case $n=2$, we can get

$$
E\left(\left(\omega_{1} h_{S} \oplus \cdots \oplus \omega_{k} h_{S}\right) \oplus \omega_{k+1} h_{S}\right)=E\left(h_{S}\right),
$$

where $\sum_{j=1}^{k+1} \omega_{j}=1$.

Second, we prove $D\left(\operatorname{EHFLWA}\left(h_{S}, h_{S}, \cdots, h_{S}\right)\right)$ $=D\left(h_{S}\right)$ in the following. The max and min linguistic terms in $h_{S}$ are denoted by $s_{\alpha^{+}}$and $s_{\alpha^{-}}$, then $D\left(h_{S}\right)=\left(\alpha^{+}-\alpha^{-}\right) /(2 t+1)$. The max virtual linguistic term in EHFLWA $\left(h_{S}, h_{S}, \cdots, h_{S}\right)$ is derived by $s_{\alpha_{1}}=\cdots=s_{\alpha_{N}}=s_{\alpha^{+}}$, then $\omega_{1} s_{\alpha_{1}} \oplus \cdots \oplus \omega_{n} s_{\alpha_{n}}=s_{\alpha^{+}}$; the min virtual linguistic term in $\operatorname{EHFLWA}\left(h_{S}, h_{S}, \cdots, h_{S}\right)$ is derived by $s_{\alpha_{1}}=\cdots=s_{\alpha_{N}}=s_{\alpha^{-}}$, then $\omega_{1} s_{\alpha_{1}} \oplus \cdots \oplus \omega_{n} s_{\alpha_{n}}=s_{\alpha^{-}}$. Thus

$D\left(\operatorname{EHFLWA}\left(h_{S}, h_{S}, \cdots, h_{S}\right)\right)=\left(\alpha^{+}-\alpha^{-}\right) /(2 t+1)=D\left(h_{S}\right)$

According to Definition 13,

$$
\operatorname{EHFLWA}\left(h_{S}, h_{S}, \cdots, h_{S}\right) \cong h_{S} \text {. }
$$

Theorem 11. (Quasi-Boundary) Let $\left\{h_{S}^{j}\right\}, j=1,2, \cdots, n$, be a set of EHFLTS. Then

$$
s_{-L} \leq \operatorname{EHFLWA}\left(h_{S}^{1}, h_{S}^{2}, \cdots, h_{S}^{n}\right) \leq s_{L},
$$

where $s_{-L}=\min _{j}\left\{\min _{s_{\alpha_{j}} \in h_{S}^{j}}\left\{s_{\alpha_{j}}\right\}\right\}, s_{L}=\max _{j}\left\{\max _{s_{\alpha_{j}} \in h_{S}^{j}}\left\{s_{\alpha_{j}}\right\}\right\}$.

\section{Proof. Since}

$$
s_{-L}=\min _{j}\left\{\min _{s_{\alpha_{j}} \in h_{s}^{h}}\left\{s_{\alpha_{j}}\right\}\right\} \leq s_{\alpha_{i}} \leq \max _{j}\left\{\max _{s_{\alpha_{j}} \in h_{s}^{j}}\left\{s_{\alpha_{j}}\right\}\right\}=s_{L}
$$

holds for $i=1,2, \cdots, n$, then

$$
s_{-L} \leq \bigoplus_{i=1}^{n} \omega_{j} s_{\alpha_{i}} \leq s_{L},
$$

which leads to

$$
s_{-L} \leq E\left(E H F L W A\left(h_{S}^{1}, h_{S}^{2}, \cdots, h_{S}^{n}\right)\right) \leq s_{L} .
$$

Thus $s_{-L} \leq \operatorname{EHFLWA}\left(h_{S}^{1}, h_{S}^{2}, \cdots, h_{S}^{n}\right) \leq s_{L}$.

\section{Group decision making with EHFLTSs}

In this section, we focus on GDM problems with the application of the proposed EHFLTSs and EHFLTs. We describe the problem mathematically at first. Then, as a solution, a new linguistic GDM model is presented. Corresponding procedures are specified for some special scenarios.

\subsection{Problem description}

As can be seen in literatures ${ }^{43-45}$, practical decision making problems with large scales of evaluations are usually done by a decision organization with several groups of experts instead of one group. A rational way to form the organization is that experts in a group have similar knowledge and experiences while experts in different group own different professional area. This organization can accumulate the advantages and alleviate the negative effects of a group (See Kang et al. 46 for detail). Therefore, in this section, as the application of the proposed technique, we focus on this kind of GDM problems, which is described mathematically as follow.

A decision organization is formed by $L$ groups (denoting by $G_{l}(l=1,2, \cdots, L)$ ) of $K_{l}$ experts denoted by $E=\left\{e_{l k} \mid k=1,2, \cdots, K_{l}, l=1,2, \cdots, L\right\}$. The weights of $L$ groups are $\omega^{(G)}=\left(\omega_{1}^{(G)}, \omega_{2}^{(G)}, \cdots, \omega_{L}^{(G)}\right)^{T}$. Usually, numbers of experts in each group are between 2 and 7 , i.e., $2 \leq K_{l} \leq 7$. The relative weights of experts within a 
group are indifferent, because of their similar backgrounds, or because of anonymity. The organization is authorized to evaluate a set of alternatives $A=\left\{a_{1}, a_{2}, \cdots, a_{M}\right\}$ in terms of a set of criteria $C=\left\{c_{1}, c_{2}, \cdots, c_{N}\right\}$. The weights of criteria are $\omega^{(C)}=\left(\omega_{1}^{(C)}, \omega_{2}^{(C)}, \cdots, \omega_{N}^{(C)}\right)^{T}$. Group $G_{l}$ evaluates a subset of $C$ in isolation, denoted by $S C_{l}$, such that $S C_{l} \neq \phi \quad, \quad l=1,2, \cdots, L \quad$ and $\bigcup_{l=1,2, \cdots, L} S C_{l}=C$. The satisfaction degree of $a_{m}$, evaluated by $e_{l k}$, with respect to criterion $c_{n}$, is represented by a function $h$ : $A \times E \times C \rightarrow S$ in linguistic setting. In this paper, the function $h$ can be expressed by HFLTSs and EHFLTSs, thus are generally denoted by EHFLTSs. Practically, experts may prefer to use linguistic term sets with distinct cardinalities. Therefore, we need to unify the multi-granular linguistic information in a unique linguistic term set before starting the process of decision making. An rational alternative is the transformation function proposed in $\mathrm{Xu}^{47}$. Suppose that the linguistic expression representing the satisfaction degree of $a_{m}$, evaluated by $e_{l k}$, with respect to criterion $c_{n}$, is transformed to a HFLTS $h_{s}^{l k}\left(a_{m}, c_{n}\right)$, where $k=1,2, \cdots, K_{l} \quad, \quad l=1,2, \cdots, L \quad, \quad m=1,2, \cdots, M \quad$, $n=1,2, \cdots, N$. The aim of the problem is to synthesize evaluation functions of each alternative and then result to a final decision.

\subsection{Group decision making model}

As seen in Fig. 3, the proposed linguistic group decision making model includes three parts.

Part 1 Structure of groups in the organization. Experts in each group are homogeneous while experts in different groups are heterogeneous. Each group deals with a part of evaluations according to its knowledge and specialty. Individuals of a group work in isolation if possible. The whole organization works collectively to complete the entire evaluations.

Part 2 Evaluation and transformation. Given a linguistic term set $S$, HFLTSs can be directly used by the experts to elicit several linguistic values for a linguistic variable when experts hesitate among several values. However, such elements are not similar to human beings' way of thinking and reasoning. Therefore, Rodriguez ${ }^{19}$ defined a context-free grammar to generate linguistic expressions that are more similar to human beings' expressions. Then the linguistic expressions provided by experts are transformed into HFLTS by using a transformation function (See Rodriguez ${ }^{19}$ for detail). According to way of individual thinking in fuzzy uncertain circumstance and the proposed construction axiom, in this model, individual evaluations are represented by linguistic expressions similar to human beings' way of thinking and reasoning and then transformed to HFLTSs.

Part 3 Synthesis for decision making. In this phase, two tools are used for synthesis. Union is used to transform HFLTSs to a generalized case, EHFLTSs. Aggregation operators are used to synthesize opinions represented by EHFLTSs. The most difference between union and aggregation operator is that all the original information are kept when the former is used while averaging value is obtained by some means when an aggregation operator is used. Thus the proposed model can reduce the use of aggregation operators and eliminate loss of information.

\subsection{Two group decision making processes}

In this section, we specify the proposed GDM model in two different scenarios with different types of weights. For simplicity, we suppose that every group is authorized to evaluate alternatives with respect to the whole set of criteria.

Scenario 1 Weights take the form of linguistic terms. Suppose $\omega_{l}^{(G)} \in S \quad, \quad l=1,2, \cdots, L \quad$ and $\quad \omega^{(C)} \in S$, $j=1,2, \cdots, N$.

Step 1: Union within each group. The evaluation information of $a_{m}$, with respect to criterion $c_{n}$, provided by group $G_{l}$, denoted by $h_{s}^{l}\left(a_{m}, c_{n}\right)$, is derived by

$$
h_{s}^{l}\left(a_{m}, c_{n}\right)=\bigcup_{k=1}^{K_{l}} h_{s}^{l k}\left(a_{m}, c_{n}\right),
$$

where $l=1,2, \cdots, L, m=1,2, \cdots, M, n=1,2, \cdots, N$.

Step 2: The collective overall preference values of $a_{m}$ with respect to criterion $c_{n}$, denoted by $h_{S}\left(a_{m}, c_{n}\right)$, are obtained by the EHFOHA operator:

$$
\begin{aligned}
& h_{S}\left(a_{m}, c_{n}\right) \\
& \quad=\operatorname{EHFOHA}\left(h_{s}^{1}\left(a_{m}, c_{n}\right), h_{s}^{2}\left(a_{m}, c_{n}\right), \cdots, h_{s}^{L}\left(a_{m}, c_{n}\right)\right)
\end{aligned}
$$

where $m=1,2, \cdots, M, \quad n=1,2, \cdots, N, \quad w=\left(w_{1}, \cdots, w_{L}\right)$ is the associated weighting vector of the EHFOHA operator with $w_{l} \in S, l=1,2, \cdots, L$. 
Step 3: The overall preference values of $a_{m}$, denoted by $h_{S}\left(a_{m}\right)$ are obtained utilizing the EHFLWD operator:

$$
\begin{aligned}
& h_{S}\left(a_{m}\right) \\
& \quad=\operatorname{EHFLWD}\left(h_{S}\left(a_{m}, c_{1}\right), h_{S}\left(a_{m}, c_{2}\right), \cdots, h_{S}\left(a_{m}, c_{N}\right)\right)
\end{aligned}
$$

where $m=1,2, \cdots, M$.

Step 4: Utilize the overall preference values $h_{S}\left(a_{m}\right)$ to rank the alternatives $a_{m}(m=1,2, \cdots, M)$, and then select the best one(s) for final decision.

Scenario 2 Weights take the form of real numbers. Suppose $\sum_{l=1}^{L} \omega_{l}^{(G)}=1, \quad \omega_{l}^{(G)} \in[0,1], l=1,2, \cdots, L$ and $\sum_{l=1}^{N} \omega_{l}^{(C)}=1, \omega_{n}^{(C)} \in[0,1], j=1,2, \cdots, N$.

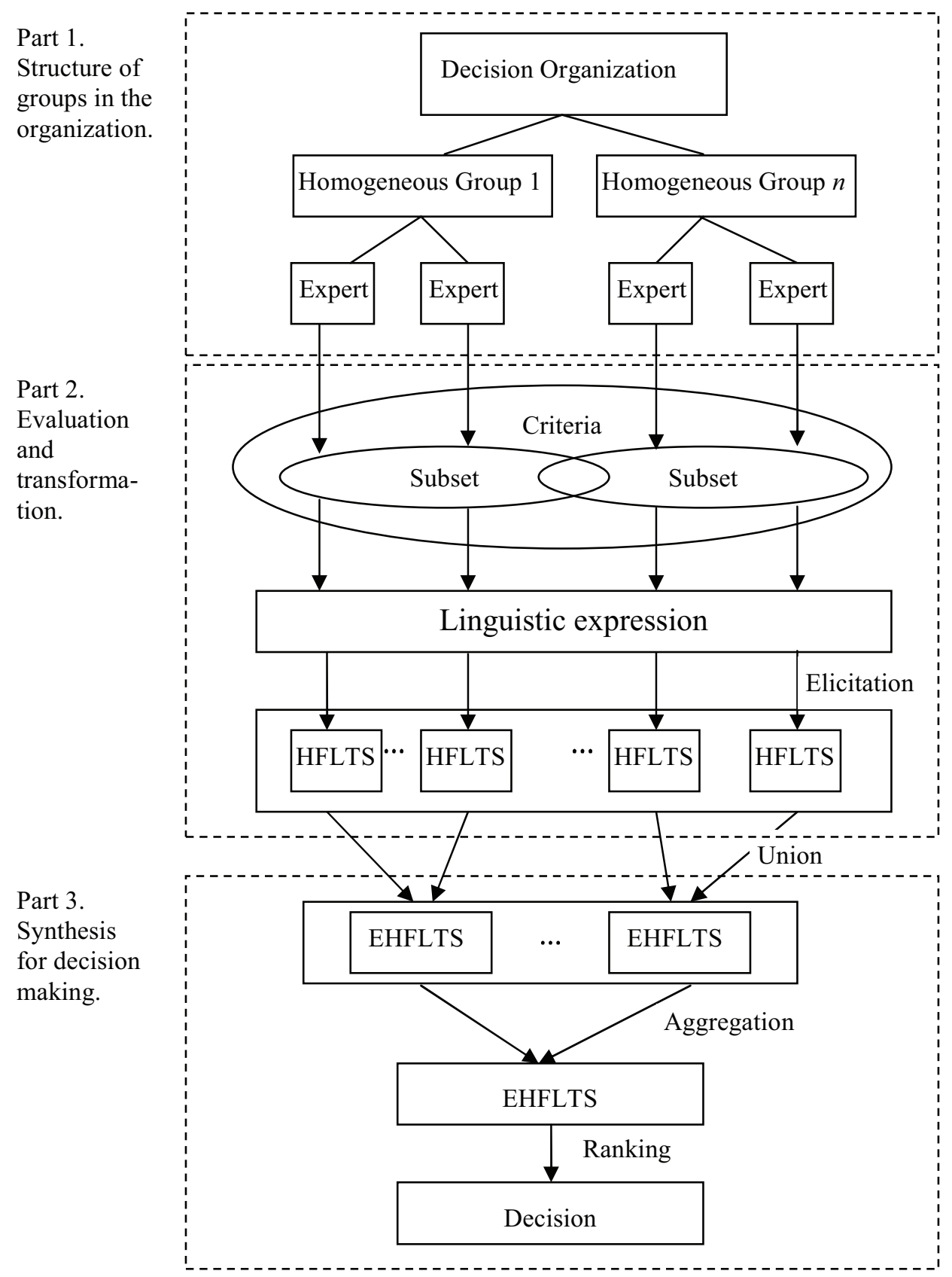

Fig. 3. Schema of the linguistic group decision making model. 
Step 1: See Step 1 of Scenario 1.

Step 2: The collective overall preference values of $a_{m}$ with respect to criterion $c_{n}$, denoted by $h_{S}\left(a_{m}, c_{n}\right)$, are obtained by the EHFLHA operator:

$$
\begin{aligned}
& h_{S}\left(a_{m}, c_{n}\right) \\
& \quad=\operatorname{EHFLHA}\left(h_{s}^{1}\left(a_{m}, c_{n}\right), h_{s}^{2}\left(a_{m}, c_{n}\right), \cdots, h_{s}^{L}\left(a_{m}, c_{n}\right)\right)
\end{aligned}
$$

where $m=1,2, \cdots, M, n=1,2, \cdots, N, w=\left(w_{1}, \cdots, w_{L}\right)$ is the associated weighting vector of the EHFLHA operator such that $\sum_{l=1}^{L} w_{l}=1, w_{l} \in[0,1], l=1,2, \cdots, L$.

Step 3: The overall preference values of $a_{m}$, denoted by $h_{S}\left(a_{m}\right)$ are obtained utilizing the EHFLWA operator:

$$
\begin{aligned}
& h_{S}\left(a_{m}\right) \\
& \quad=\operatorname{EHFLWA}\left(h_{S}\left(a_{m}, c_{1}\right), h_{S}\left(a_{m}, c_{2}\right), \cdots, h_{S}\left(a_{m}, c_{N}\right)\right)
\end{aligned}
$$

where $m=1,2, \cdots, M$.

Step 4: See Step 4 of Scenario 1.

We assume that the weighting vectors are completely known in above scenarios. In fact, if the weighting vector of criteria is absolutely unknown, we can use the corresponding OWA operator instead of weighted averaging operator. That is, in Step 3 of the two scenarios, we can replace the EHFLWD operator and the EHFLWA operator with the EHFOOWA operator and the EHFLOWA operator respectively. Further, the associated weighting vector of the EHFLOWA operator can be determined by the normal distribution based method or others ${ }^{48}$.

\section{Application and Discussion}

In this section, we apply the proposed linguistic GDM model and processes in a practical example. Then, some discussions are given to compare the proposed technique with some existing approaches.

\subsection{An example}

A practical GDM problem of evaluating university faculty for tenure and promotion ${ }^{41,49}$ is used to illustrate the proposed processes. The criteria used in some universities are $c_{1}$ : teaching, $c_{2}$ : research, and $c_{3}$ : service (whose weighting vector is $\left.\omega^{(C)}=(0.14,0.26,0.60)^{T}\right)$. Five alternatives (faculty candidates $) a_{m}(m=1,2,3,4,5)$ are to be evaluated using the linguistic term set $S$ in Example 2 by two groups of experts (whose weighting vector
$\left.\omega^{(G)}=(0.60,0.40)^{T}\right)$. The first group $G_{1}$ includes 3 experts $e_{11}, e_{12}, e_{13}$, and the second group $G_{2}$ is formed by 2 experts $e_{21}, e_{22}$. After elicitation, experts' evaluation information is listed in Tables 1-5.

As the weighting vectors take the form of real number, we utilize the process of Scenario 2 to meet a decision.

Step 1: The evaluation information of $a_{m}$ ( $m=1,2,3,4,5)$, with respect to criterion $c_{n}(n=1,2,3)$, provided by group $G_{1}$ and $G_{2}$, are derived by the union of HFLTSs provided by experts of each group. The results are listed in Tables 6-7.

Step 3: The overall preference values of $a_{m}$, denoted by $h_{S}\left(a_{m}\right), m=1,2,3,4,5$, are obtained utilizing the EHFLWA operator. As the cardinality of $h_{S}\left(a_{m}\right)$ is huge, only the expected linguistic terms are given:

Table 1. Decision matrix provided by $e_{11}$.

\begin{tabular}{llllll}
\hline$c_{n}$ & $a_{1}$ & $a_{2}$ & $a_{3}$ & $a_{4}$ & $a_{5}$ \\
\hline$c_{1}$ & $\left\{s_{-1}, s_{0}\right\}$ & $\left\{s_{-2}\right\}$ & $\left\{s_{-1}, s_{0}\right\}$ & $\left\{s_{2}\right\}$ & $\left\{s_{1}, s_{2}\right\}$ \\
$c_{2}$ & $\left\{s_{0}\right\}$ & $\left\{s_{1}, s_{2}\right\}$ & $\left\{s_{-2}\right\}$ & $\left\{s_{1}, s_{2}\right\}$ & $\left\{s_{1}\right\}$ \\
$c_{3}$ & $\left\{s_{2}\right\}$ & $\left\{s_{2}, s_{3}\right\}$ & $\left\{s_{2}\right\}$ & $\left\{s_{-1}\right\}$ & $\left\{s_{-1}, s_{0}\right\}$ \\
\hline
\end{tabular}

Table 2. Decision matrix provided by $e_{12}$.

\begin{tabular}{llllll}
\hline$c_{n}$ & $a_{1}$ & $a_{2}$ & $a_{3}$ & $a_{4}$ & $a_{5}$ \\
\hline$c_{1}$ & $\left\{s_{-1}\right\}$ & $\left\{s_{-2}, s_{0}\right\}$ & $\left\{s_{-1}\right\}$ & $\left\{s_{2}\right\}$ & $\left\{s_{-1}\right\}$ \\
$c_{2}$ & $\left\{s_{2}, s_{3}\right\}$ & $\left\{s_{1}\right\}$ & $\left\{s_{-2}\right\}$ & $\left\{s_{1}\right\}$ & $\left\{s_{1}, s_{2}\right\}$ \\
$c_{3}$ & $\left\{s_{2}\right\}$ & $\left\{s_{1}, s_{2}\right\}$ & $\left\{s_{2}\right\}$ & $\left\{s_{1}\right\}$ & $\left\{s_{2}\right\}$ \\
\hline
\end{tabular}

Table 3. Decision matrix provided by $e_{13}$.

\begin{tabular}{llllll}
\hline$c_{n}$ & $a_{1}$ & $a_{2}$ & $a_{3}$ & $a_{4}$ & $a_{5}$ \\
\hline$c_{1}$ & $\left\{s_{-1}, s_{0}\right\}$ & $\left\{s_{0}\right\}$ & $\left\{s_{0}, s_{1}\right\}$ & $\left\{s_{2}, s_{3}\right\}$ & $\left\{s_{-1}, s_{1}\right\}$ \\
$c_{2}\left\{s_{0}\right\}$ & $\left\{s_{1}, s_{2}\right\}$ & $\left\{s_{-2}\right\}$ & $\left\{s_{2}\right\}$ & $\left\{s_{1}\right\}$ \\
$c_{3}$ & $\left\{s_{0}\right\}$ & $\left\{s_{1}, s_{2}\right\}$ & $\left\{s_{2}\right\}$ & $\left\{s_{-1}\right\}$ & $\left\{s_{-1}, s_{0}\right\}$ \\
\hline
\end{tabular}

Table 4. Decision matrix provided by $e_{21}$.

\begin{tabular}{llllll}
\hline$c_{n}$ & $a_{1}$ & $a_{2}$ & $a_{3}$ & $a_{4}$ & $a_{5}$ \\
\hline$c_{1}$ & $\left\{s_{0}\right\}$ & $\left\{s_{1}, s_{2}\right\}$ & $\left\{s_{1}\right\}$ & $\left\{s_{2}\right\}$ & $\left\{s_{-1}\right\}$ \\
$c_{2}$ & $\left\{s_{1}, s_{2}\right\}$ & $\left\{s_{1}\right\}$ & $\left\{s_{0}, s_{1}\right\}$ & $\left\{s_{1}, s_{2}\right\}$ & $\left\{s_{2}\right\}$ \\
$c_{3}$ & $\left\{s_{0}\right\}$ & $\left\{s_{3}\right\}$ & $\left\{s_{2}\right\}$ & $\left\{s_{2}\right\}$ & $\left\{s_{2}\right\}$ \\
\hline
\end{tabular}


Table 5. Decision matrix provided by $e_{22}$.

\begin{tabular}{llllll}
\hline$c_{n}$ & $a_{1}$ & $a_{2}$ & $a_{3}$ & $a_{4}$ & $a_{5}$ \\
\hline$c_{1}$ & $\left\{s_{2}\right\}$ & $\left\{s_{2}\right\}$ & $\left\{s_{0}, s_{1}\right\}$ & $\left\{s_{2}\right\}$ & $\left\{s_{2}\right\}$ \\
$c_{2}$ & $\left\{s_{2}, s_{3}\right\}$ & $\left\{s_{1}\right\}$ & $\left\{s_{-2}\right\}$ & $\left\{s_{1}\right\}$ & $\left\{s_{2}\right\}$ \\
$c_{3}$ & $\left\{s_{1}\right\}$ & $\left\{s_{0}\right\}$ & $\left\{s_{1}\right\}$ & $\left\{s_{-1}, s_{0}\right\}$ & $\left\{s_{-1}, s_{0}\right\}$ \\
\hline
\end{tabular}

$$
\begin{gathered}
E\left(h_{S}\left(a_{1}\right)\right)=s_{0.7970}, E\left(h_{S}\left(a_{2}\right)\right)=s_{1.5860}, \\
E\left(h_{S}\left(a_{3}\right)\right)=s_{0.7053}, E\left(h_{S}\left(a_{4}\right)\right)=s_{0.7920}, \\
E\left(h_{S}\left(a_{5}\right)\right)=s_{0.8860} .
\end{gathered}
$$

Step 4: According to Definition 13, we can obtain that:

$$
h_{S}\left(a_{3}\right)<h_{S}\left(a_{4}\right)<h_{S}\left(a_{1}\right)<h_{S}\left(a_{5}\right)<h_{S}\left(a_{2}\right),
$$

thus, the order of these faculty candidates is

$$
a_{2}>a_{5}>a_{1}>a_{4}>a_{3},
$$

then $a_{2}$ is the best candidate.

\subsection{Comparison and Discussion}

As an alternative solution of the above problem, the LA operator ${ }^{40}$, the LWA operator and LHA operator are used for comparison. Without the idea of HFS, we cannot deal with several possible values at the same

Table 6. Decision matrix of $G_{1}$.

\begin{tabular}{|c|c|c|c|}
\hline$a_{m}$ & $c_{1}$ & $c_{2}$ & $c_{3}$ \\
\hline$a_{1}$ & $\begin{array}{l}\left\{\left(s_{-1}, .4\right),\left(s_{0}, 0\right),\left(s_{0}, .2\right),\right. \\
\left.\left(s_{1},-.2\right)\right\}\end{array}$ & $\begin{array}{l}\left\{\left(s_{0}, .4\right),\left(s_{1},-.2\right),\left(s_{1}, .2\right),\right. \\
\left(s_{2},-.4\right),\left(s_{2}, 0\right),\left(s_{2}, .2\right), \\
\left.\left(s_{2}, .4\right),\left(s_{3},-.4\right),\left(s_{3}, 0\right)\right\}\end{array}$ & $\begin{array}{l}\left\{\left(s_{0}, 0\right),\left(s_{0}, .4\right),\left(s_{1}, .2\right),\right. \\
\left.\left(s_{2},-.4\right)\right\}\end{array}$ \\
\hline$a_{2}$ & $\begin{array}{l}\left\{\left(s_{0}, .4\right),\left(s_{1},-.2\right),\left(s_{2},-.4\right),\right. \\
\left.\left(s_{2}, 0\right)\right\}\end{array}$ & $\left\{\left(s_{1}, 0\right),\left(s_{2},-.4\right)\right\}$ & $\begin{array}{l}\left\{\left(s_{1},-.4\right),\left(s_{1}, .2\right),\left(s_{2},-.2\right),\right. \\
\left.\left(s_{2}, .4\right),\left(s_{3}, 0\right)\right\}\end{array}$ \\
\hline$a_{3}$ & $\begin{array}{l}\left\{\left(s_{-1}, 0\right),\left(s_{-1}, .4\right),\left(s_{0},-.4\right),\right. \\
\left.\left(s_{0}, 0\right),\left(s_{0}, .2\right),\left(s_{1},-.4\right)\right\}\end{array}$ & $\begin{array}{l}\left\{\left(s_{-2}, 0\right),\left(s_{-1},-.2\right),\left(s_{-1}, .2\right)\right. \\
\}\end{array}$ & $\left\{\left(s_{2},-.4\right),\left(s_{2}, 0\right)\right\}$ \\
\hline$a_{4}$ & $\left\{\left(s_{2}, 0\right),\left(s_{3},-.4\right)\right\}$ & $\begin{array}{l}\left\{\left(s_{1}, 0\right),\left(s_{1}, .4\right),\left(s_{2},-.4\right),\right. \\
\left.\left(s_{2}, 0\right)\right\}\end{array}$ & $\begin{array}{l}\left\{\left(s_{-1}, 0\right),\left(s_{-1}, .4\right),\left(s_{0}, .2\right),\right. \\
\left.\left(s_{1},-.4\right),\left(s_{1}, 4\right)\right\}\end{array}$ \\
\hline$a_{5}$ & $\begin{array}{l}\left\{\left(s_{-1}, 0\right),\left(s_{0}, .2\right),\left(s_{1},-.2\right),\right. \\
\left.\left(s_{1}, 4\right),\left(s_{2}, 0\right)\right\}\end{array}$ & $\left\{\left(s_{1}, 4\right),\left(s_{2}, 0\right)\right\}$ & $\begin{array}{l}\left\{\left(s_{-1}, 0\right),\left(s_{0},-.4\right),\left(s_{0},-.2\right),\right. \\
\left(s_{0}, .4\right),\left(s_{1},-.4\right),\left(s_{1},-.2\right), \\
\left.\left(s_{1}, .2\right),\left(s_{2},-.4\right),\left(s_{2}, .4\right)\right\}\end{array}$ \\
\hline
\end{tabular}

\begin{tabular}{llllll}
\hline$c_{n}$ & $a_{1}$ & $a_{2}$ & $a_{3}$ & $a_{4}$ & $a_{5}$ \\
\hline$c_{1}$ & $\left\{s_{-1}, s_{0}\right\}$ & $\left\{s_{-2}, s_{0}\right\}$ & $\left\{s_{-1}, s_{0}, s_{1}\right\}$ & $\left\{s_{2}, s_{3}\right\}$ & $\left\{s_{-1}, s_{1}, s_{2}\right\}$ \\
$c_{2}$ & $\left\{s_{0}, s_{2}, s_{3}\right\}$ & $\left\{s_{1}, s_{2}\right\}$ & $\left\{s_{-2}\right\}$ & $\left\{s_{1}, s_{2}\right\}$ & $\left\{s_{1}, s_{2}\right\}$ \\
$c_{3}$ & $\left\{s_{0}, s_{2}\right\}$ & $\left\{s_{1}, s_{2}, s_{3}\right\}$ & $\left\{s_{2}\right\}$ & $\left\{s_{-1}, s_{1}\right\}$ & $\left\{s_{-1}, s_{0}, s_{2}\right\}$ \\
\hline
\end{tabular}

Table 7. Decision matrix of $G_{2}$.

\begin{tabular}{llllll}
\hline$c_{n}$ & $a_{1}$ & $a_{2}$ & $a_{3}$ & $a_{4}$ & $a_{5}$ \\
\hline$c_{1}$ & $\left\{s_{-2}, s_{0}\right\}$ & $\left\{s_{1}, s_{2}\right\}$ & $\left\{s_{0}, s_{1}\right\}$ & $\left\{s_{2}\right\}$ & $\left\{s_{-1}, s_{2}\right\}$ \\
$c_{2}$ & $\left\{s_{1}, s_{2}, s_{3}\right\}$ & $\left\{s_{1}\right\}$ & $\left\{s_{-2}, s_{0}, s_{1}\right\}$ & $\left\{s_{1}, s_{2}\right\}$ & $\left\{s_{2}\right\}$ \\
$c_{3}$ & $\left\{s_{0}, s_{1}\right\}$ & $\left\{s_{0}, s_{3}\right\}$ & $\left\{s_{1}, s_{2}\right\}$ & $\left\{s_{-1}, s_{0}, s_{2}\right\}$ & $\left\{s_{-1}, s_{1}, s_{3}\right\}$ \\
\hline
\end{tabular}

Table 8. The final decision matrix. 
time. Thus if experts have hesitancy among several possible linguistic terms, a pre-aggregation step has to be done, and then an averaging value is computed by some means. Following the advice of $\mathrm{Xu}^{41}$, the problem can be processed by some steps.

Step 1: If an expert has hesitancy among several possible linguistic terms, the LA operator is used to obtain the corresponding averaging values. For example, the resultant decision matrix provided by $e_{11}$ is transformed into Table 9.

Table 9. Decision matrix provided by $e_{11}$ after preaggregation.

\begin{tabular}{llllll}
\hline$c_{n}$ & $a_{1}$ & $a_{2}$ & $a_{3}$ & $a_{4}$ & $a_{5}$ \\
\hline$c_{1}$ & $\left(s_{0},-.5\right)$ & $\left(s_{-2}, 0\right)$ & $\left(s_{0},-.5\right)$ & $\left(s_{2}, 0\right)$ & $\left(s_{2},-.5\right)$ \\
$c_{2}$ & $\left(s_{0}, 0\right)$ & $\left(s_{2},-.5\right)$ & $\left(s_{-2}, 0\right)$ & $\left(s_{2},-.5\right)$ & $\left(s_{1}, 0\right)$ \\
$c_{3}$ & $\left(s_{2}, 0\right)$ & $\left(s_{3},-.5\right)$ & $\left(s_{2}, 0\right)$ & $\left(s_{-1}, 0\right)$ & $\left(s_{0},-.5\right)$ \\
\hline
\end{tabular}

Step 2: The evaluations within each group are synthesized by the LA operator as well as the relative weights of experts within a group are indifferent. The resultant decision matrices are shown in Tables 10 and 11.

Step 3: We aggregate Tables 10 and 11 by the LHA operator to obtain the final decision matrix. The weighting vector and associated weighting vector are the same as used in Section 6.1. The result is presented in Table 12.
Step 4: The overall satisfactory degree of $a_{m}$, denoted by $z_{m}, m=1,2,3,4,5$, are obtained utilizing the LWA operator as follows:

$$
\begin{gathered}
z_{1}=\left(s_{1},-.062\right), z_{2}=\left(s_{1}, .19\right), z_{3}=\left(s_{1},-296\right), \\
z_{4}=\left(s_{1},-.042\right), z_{5}=\left(s_{1},-.268\right) .
\end{gathered}
$$

Therefore, $z_{2}>z_{4}>z_{1}>z_{5}>z_{3}$.

Step 5: The alternatives can be ranked as:

$$
a_{2}>a_{4}>a_{1}>a_{5}>a_{3} \text {. }
$$

Based on the procedures of the comparable processes, we discuss their differences by the following aspects.

1) Number of times of using aggregation operators. As the idea of HFS is used, the proposed model and process need less aggregation. Comparing to the existing process, the pre-aggregation step in expert level is eliminated. Further, aggregation within each group is conducted by the union operation rather than an aggregation operator.

2) Possible values versus averaging values. Because of less aggregation operator is used, all possible values, rather than only averaging values, are maintained for consideration. As in Table 1 and Table 9, the proposed process use HFLTSs to represent experts' opinion, while existing process has to synthesize all possible linguistic terms to an averaging value. The same phenomenon happens in each level of aggregation. Therefore, the proposed process handle all possible values along with the procedure of aggregation, it is more meaningful than considering just averaging values as there is no loss of information.

Table 10. Decision matrix of $G_{1}$ after second aggregation.

\begin{tabular}{llllll}
\hline$c_{n}$ & $a_{1}$ & $a_{2}$ & $a_{3}$ & $a_{4}$ & $a_{5}$ \\
\hline$c_{1}$ & $\left(s_{-1}, .3333\right)$ & $\left(s_{-1}, 0\right)$ & $\left(s_{0},-.3333\right)$ & $\left(s_{2}, .1667\right)$ & $\left(s_{0}, .1667\right)$ \\
$c_{2}$ & $\left(s_{1},-.1667\right)$ & $\left(s_{1}, .3333\right)$ & $\left(s_{-2}, 0\right)$ & $\left(s_{2},-.5\right)$ & $\left(s_{1}, .1667\right)$ \\
$c_{3}$ & $\left(s_{1}, .3333\right)$ & $\left(s_{2},-.1667\right)$ & $\left(s_{2}, 0\right)$ & $\left(s_{0}, .3333\right)$ & $\left(s_{0}, .3333\right)$ \\
\hline
\end{tabular}

Table 11. Decision matrix of $G_{2}$ after second aggregation.

\begin{tabular}{llllll}
\hline$c_{n}$ & $a_{1}$ & $a_{2}$ & $a_{3}$ & $a_{4}$ & $a_{5}$ \\
\hline$c_{1}$ & $\left(s_{1}, 0\right)$ & $\left(s_{2},-.25\right)$ & $\left(s_{1},-.25\right)$ & $\left(s_{2}, 0\right)$ & $\left(s_{1},-.5\right)$ \\
$c_{2}$ & $\left(s_{2}, 0\right)$ & $\left(s_{1}, 0\right)$ & $\left(s_{-1}, .25\right)$ & $\left(s_{1}, .25\right)$ & $\left(s_{2}, 0\right)$ \\
$c_{3}$ & $\left(s_{1},-.5\right)$ & $\left(s_{2},-.5\right)$ & $\left(s_{2},-.5\right)$ & $\left(s_{1},-.25\right)$ & $\left(s_{1},-.25\right)$ \\
\hline
\end{tabular}

Table 12. The final decision matrix after third aggregation.

\begin{tabular}{llllll}
\hline$c_{n}$ & $a_{1}$ & $a_{2}$ & $a_{3}$ & $a_{4}$ & $a_{5}$ \\
\hline$c_{1}$ & $\left(s_{0}, 0\right)$ & $\left(s_{0}, .10\right)$ & $\left(s_{0}, .10\right)$ & $\left(s_{2}, .10\right)$ & $\left(s_{0}, .30\right)$ \\
$c_{2}$ & $\left(s_{1}, .30\right)$ & $\left(s_{1},-.40\right)$ & $\left(s_{0},-.50\right)$ & $\left(s_{1}, .40\right)$ & $\left(s_{2},-.50\right)$ \\
$c_{3}$ & $\left(s_{1}, 0\right)$ & $\left(s_{2},-.30\right)$ & $\left(s_{2},-.20\right)$ & $\left(s_{0},-.50\right)$ & $\left(s_{1},-.50\right)$ \\
\hline
\end{tabular}


3) Final decision. Both methods are different while agree on the first choice $a_{2}$, which validates that the proposed process is reasonable and it is useful to consider all possible values. . We can also see that the priorities of five alternatives are distinct. There is a rank reversal between $a_{4}$ and $a_{5}$. The proposed process uses all possible values for synthesis and need less aggregation, as analyzed above, thus the final decision would be more rational.

\section{Conclusion remarks}

In this paper, we have extended HFLTSs to EHFLTSs for the purpose of application. Some basic operations and two types of aggregation operators with distinct forms of weighting vector have been proposed. EHFLTSs have more desirable mathematical properties than HFLTS. The construction axiom and linguistic GDM model have been presented for the potential application of the proposed EHFLTSs. At last, a practical example has shown that the proposed technique owns several advantages and obtains more rational decision than the existing method.

Using the proposed EHFLTS and the proposed model, we can take all possible values into account during the procedure of information aggregation. At the same time, less aggregation operators are needed, which makes final decision more robust because more aggregation may lead to less robust decision ${ }^{9}$. Furthermore, as linguistic term sets and HFLTSs can be seen as special cases of the proposed set, EHFLTSs can model more complex and complicated decision making problems in linguistic setting.

As future work we will develop some more aggregation operators, such as induced OWA operators and generalized OWA operators ${ }^{50}$ to support the proposed model. Solutions of more general cases of the proposed model will be also considered. Some measures of EHFLTSs used for clustering and data mining, such as degree of similarity, distance and so on, are also challenges.

\section{Acknowledgments}

The author would like to thank the editor and two reviewers for various helpful and fruitful comments that led to many significant results.

\section{References}

1. Shafer G. A mathematical theory of evidence: Princeton university press Princeton; 1976.

2. Yang JB, Xu DL. On the evidential reasoning algorithm for multiple attribute decision analysis under uncertainty. IEEE Transactions on Systems, Man and Cybernetics, Part A: Systems and Humans 2002;32(3):289-304.

3. Zadeh LA. Fuzzy sets. Information Control 1965;8:338353.

4. Mizumoto M, Tanaka K. Some properties of fuzzy sets of type 2. Information and control 1976;31(4):312-340.

5. D. D, Prade H. Fuzzy Sets and Systems: Theory and Applications. New York: Kluwer Academic; 1980.

6. Atanassov KT. Intuitionistic fuzzy sets. Fuzzy Sets and Systems 1986;20(1):87-96.

7. RONALD RY. On the theory of bags. International Journal Of General System 1986;13(1):23-37.

8. Torra V. Hesitant Fuzzy Sets. International Journal of Intelligent Systems 2010;25(6):529-539.

9. Qian G, Wang H, Feng X-q. Generalization Hesitant Fuzzy Sets and their application in decision support systems. Knowledge-Based Systems 2013;37(1):357-365.

10. $\mathrm{Xu} \mathrm{Z}$. Linguistic aggregation operators: an overview. Fuzzy sets and their extensions: representation, aggregation and models 2008:163-181.

11. Zadeh LA. The concept of a linguistic variable and its application to approximate reasoning--I. Information Sciences 1975;8(3):199-249.

12. Yager RR. On the retranslation process in Zadeh's paradigm of computing with words. Systems, Man, and Cybernetics, Part B: Cybernetics, IEEE Transactions on 2004;34(2):1184-1195.

13. Türkşen IB. Type 2 representation and reasoning for CWW. Fuzzy Sets and Systems 2002;127(1):17-36.

14. Yager RR. A new methodology for ordinal multiobjective decision based on fuzzy sets. Decision Sciences 1981;12(4):589-600.

15. Delgado M, Verdegay JL, Vila M. On aggregation operations of linguistic labels. International Journal of Intelligent Systems 1993;8(3):351-370.

16. $\mathrm{Xu} \mathrm{Z}$. A method based on linguistic aggregation operators for group decision making with linguistic preference relations. Information Sciences 2004;166(1):19-30.

17. Herrera F, Martinez L. A 2-tuple fuzzy linguistic representation model for computing with words. Fuzzy Systems, IEEE Transactions on 2000;8(6):746-752.

18. Wang JH, Hao J. A new version of 2-tuple fuzzy linguistic representation model for computing with words. IEEE Transactions on Fuzzy Systems 2006;14(3):435445.

19. Rodriguez RM, Martinez L, Herrera F. Hesitant Fuzzy Linguistic Term Sets for Decision Making. IEEE Transactions on Fuzzy Systems 2012;20(1):109-119. 
20. Rodríguez RM, Martínez L, Herrera F. A group decision making model dealing with comparative linguistic expressions based on hesitant fuzzy linguistic term sets. Information Sciences 2013;241(0):28-42.

21. $\mathrm{Xu} \mathrm{ZS.} \mathrm{EOWA} \mathrm{and} \mathrm{EOWG} \mathrm{operators} \mathrm{for} \mathrm{aggregating}$ linguistic labels based on linguistic preference relations. International Journal of Uncertainty Fuzziness and Knowledge-Based Systems 2004;12:791-810.

22. Torra V. Negation functions based semantics for ordered linguistic labels. International Journal of Intelligent Systems 1996;11(11):975-988.

23. Zadeh LA. The concept of a linguistic variable and its application to approximate reasoning-I. Information Sciences 1975;8(3):199-249.

24. Zadeh LA. The concept of a linguistic variable and its application to approximate reasoning-II. Information Sciences 1975;8(4):301-357.

25. Zadeh LA. The concept of a linguistic variable and its application to approximate reasoning-III. Information Sciences 1975;9(1):43-80.

26. Herrera F, Herrera-Viedma E, Verdegay JL. A sequential selection process in group decision making with a linguistic assessment approach. Information Sciences 1995;85(4):223-239.

27. Bordogna G, Fedrizzi M, Pasi G. A linguistic modeling of consensus in group decision making based on OWA operators. IEEE Transactions on Systems, Man and Cybernetics, Part A: Systems and Humans 1997;27(1):126-133.

28. Herrera F, Herrera-Viedma E. Linguistic decision analysis: steps for solving decision problems under linguistic information. Fuzzy Sets and Systems 2000;115(1):67-82.

29. $\mathrm{Xu} \mathrm{Z}$. Deviation measures of linguistic preference relations in group decision making. Omega 2005;33(3): 249-254.

30. Xu Z. A method for multiple attribute decision making with incomplete weight information in linguistic setting. Knowledge-Based Systems 2007;20(8):719-725.

31. Rodríguez RM, Martínez L. An analysis of symbolic linguistic computing models in decision making. International Journal of General Systems 2012;42(1):121136.

32. Martınez L, Ruan D, Herrera F. Computing with words in decision support systems: An overview on models and applications. International Journal of Computational Intelligence Systems 2010;3(4):382-395.

33. Dong $\mathrm{Y}, \mathrm{Xu} \mathrm{Y}$, Li H, Feng B. The OWA-based consensus operator under linguistic representation models using position indexes. European Journal of Operational Research 2010;203(2):455-463.

34. Xu ZS, Xia MM. Hesitant fuzzy information aggregation in decision making. International Journal of Approximate Reasoning 2011;52(3):395-407.
35. Torra V, Narukawa Y. On Hesitant Fuzzy Sets and Decision; 2009. 1378-1382.

36. Herrera F, Herrear-Viedma E. Aggregation operators for linguistic weighted information. IEEE Transactions on Systems, Man and Cybernetics-Part A 1997;27:646-656.

37. Yager RR. On ordered weighted averaging aggregation operators in multi-criteria decision making. IEEE Transactions on Systems, Man and Cybernetics 1988;18:183-190.

38. Yager RR. Applications and extensions of OWA aggregations. International Journal of Man-Machine Studied 1992;37:103-132.

39. $\mathrm{Xu}$ ZS. Uncertain multiple attribute decision making: methods and applications. Beijing: Tsinghua University Press; 2004.

40. $\mathrm{Xu}$ ZS. On generalized induced linguistic aggregation operators. International Journal of General System 2006;35:17-28.

41. Xu ZS. A note on linguistic hybrid arithmetic averaging operator in multiple attribute group decision making with linguistic information. Group Decision and Negotiation 2006;15(6):593-604.

42. Xu ZS, Da QL. An overview of operators for aggregating information. International Journal of Intelligent Systems 2003;18(9):953-969.

43. Azzi AE. Group representation and procedural justice in multigroup decision-making bodies. Social Justice Research 1993;6(2):195-218.

44. Chen TY. Optimistic and pessimistic decision making with dissonance reduction using interval-valued fuzzy sets. Information Sciences 2011;181(3):479-502.

45. Xia M, Xu Z, Chen N. Some Hesitant Fuzzy Aggregation Operators with Their Application in Group Decision Making. Group Decision and Negotiation 2011:1-21.

46. Kang L, Qian G, Wang H. Multi Groups Decision Making using Generalized Hesitant Fuzzy Sets. Information Sciences; 2014, submitted.

47. $\mathrm{Xu} \mathrm{Z}$. An Interactive Approach to Multiple Attribute Group Decision Making with Multigranular Uncertain Linguistic Information. Group Decision and Negotiation 2009;18(2):119-145.

48. Xu ZS. An overview of methods for determining OWA weights. International Journal of Intelligent Systems 2005;20(8):843-865.

49. Bryson N, Mobolurin A. An Action Learning Evaluation Procedure for Multiple Criteria Decision Making Problems. European Journal of Operational Research 1995;96(379-386).

50. Schaefer PA, Mitchell HB. A generalized OWA operator. International Journal of Intelligent Systems 1999;14(2):123-143. 